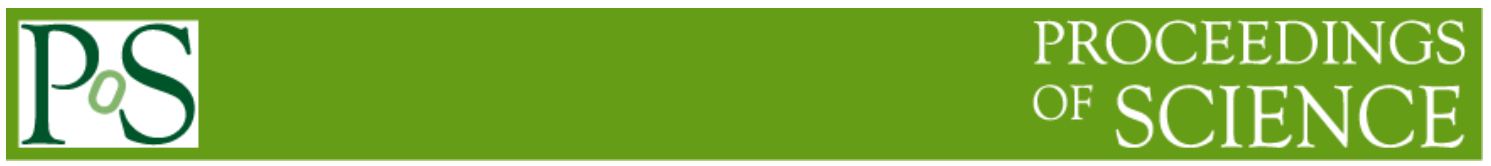

\title{
Dust in the Solar System: Properties and Origins
}

\author{
Scott Messenger ${ }^{1}$ \\ Robert M. Walker Laboratory for Space Science, \\ Astromaterials Research and Exploration Science Directorate, \\ NASA Johnson Space Center, Houston, TX, USA \\ E-mail: scott.r.messenger@nasa.gov \\ Lindsay P. Keller \\ Robert M. Walker Laboratory for Space Science \\ Astromaterials Research and Exploration Science Directorate \\ NASA Johnson Space Center, Houston, TX, USA \\ E-mail: Lindsay.p.keller@nasa.gov
}

\section{Ann N. Nguyen}

Jacobs

NASA Johnson Space Center

Houston, TX 77058, USA

E-mail: lan-anh.n.nguyen@nasa.gov

The Life Cycle of Dust in the Universe: Observations, Theory, and Laboratory Experiments (LCDU 2013

)

November 18-22, 2013

Taipei, Taiwan

1

Speaker 


\section{Introduction}

The Solar System's earliest beginnings can be traced back to the condensation of dust in the outflows of dying stars. Together with grains formed in molecular clouds, these smoke-sized particles comprised the original building blocks of the Solar System. Diverse environments within the solar nebula itself were also prodigious sources of dust, ranging from refractory minerals condensing from high temperature gas near the Sun to organic grains forming under cryogenic conditions at the outer regions of the disk. Remarkably, dust grains from all of these environments have survived intact in comets and asteroids that have undergone minimal parent body processing since their formation $\sim 4.57$ billion years ago. Today, the Solar System is suffused with a new generation of dust that is formed by mutual collisions among asteroids, by impacts onto other airless bodies, by comets entering the inner Solar System, and by a diffuse, high velocity interstellar dust stream.

Knowledge of the origins and properties of dust in the Solar System has been obtained through a combination of astronomical observations, spacecraft [1-5], and by direct laboratory analysis of samples collected on and above the Earth and in space. Here we summarize the properties and origins of present day interplanetary dust and of the Solar System starting materials as derived from laboratory analysis.

\subsection{Modern interplanetary dust}

Dust in the Solar System is visible with the naked eye as a faint glow on the horizon just following sunset or before sunrise. This 'zodiacal light' is caused by reflection of sunlight off of countless interplanetary dust particles in the inner Solar System. Interplanetary dust principally derives from mutual collisions among asteroids and as a consequence of volatile loss from comets as they approach the Sun. It is still not known with certainty whether comets or asteroids are the dominant sources of interplanetary dust.

Early attempts to discern the origin of interplanetary dust favored comets as the dominant source. Whipple [6] proposed that comet 1P/Encke alone could have contributed the majority of the dust in the Solar System. Later, IRAS observations of the Zodiacal dust revealed distinct bands whose inclination distributions were associated with several major asteroid families [7], and these observations were taken to favor asteroidal sources for most of the interplanetary dust. More recent models of the zodiacal dust cloud suggest that as much as $90 \%$ of the dust in the inner Solar System derives from comets [8]. Unfortunately, observational limitations leave large uncertainties in models for the origin of the interplanetary dust complex. Furthermore, the origins of interplanetary dust particles probably also vary with time and according to their size and location in the Solar System.

The Earth accretes interplanetary dust $(<100 \mu \mathrm{m})$ and micrometeorites $(<1 \mathrm{~mm})$ from virtually all sources $>1$ AU from the Sun. Due to a combination of Poynting-Robertson (PR) drag and solar wind drag, all particles $<1 \mathrm{~cm}$ gradually spiral into the Sun over $10^{4}-10^{5}$ year 
timescales [9]. The Poynting-Robertson effect is a relativistic drag force that arises from the anisotropic absorption and emission of radiation from small particles in motion [10, 11]. While most of the particles are ultimately captured by the Sun, some are destroyed in grain-grain collisions, captured by other Solar System bodies or are ejected from the Solar System during planetary encounters. Consequently, interplanetary dust accreted by the Earth is a more representative sample of Solar System bodies than meteorites, which mainly derive from locations in the asteroid belt near mean-motion resonances with Jupiter and rarely from the moon and Mars.

Samples of cosmic dust have been collected in especially clean locations on the surface of the Earth, in the upper atmosphere, and by satellites in low-Earth orbit. Measurements of micrometeoroid impact craters on the LDEF spacecraft have yielded the most accurate measurement of the terrestrial accretion rate of dust, approximately $4 \times 10^{7} \mathrm{~kg}$ of particles $<300$ $\mu \mathrm{m}$ in diameter every year [12]. Two spacecraft have also returned cosmic dust directly from their source bodies: the NASA Stardust mission to comet 81P/Wild 2 [13] and the Japanese (JAXA) Hayabusa mission to the near-Earth asteroid Itokawa [14]. An interplanetary dust collector deployed on the international space station has also been used to collect meteoroids just before impacting the Earth [15].

The study of the Stardust cometary samples is highly challenging because they are diverse, intimately mixed with aerogel (the capture medium), and have experienced varying degrees of thermal and shock alteration as a result of the impact into the dust collector at $6 \mathrm{~km} / \mathrm{s}$. The high velocity dust collection also resulted in the destruction and alteration of fine grained and thermally labile materials. Nevertheless, analysis of these samples revealed a number of surprising findings, including the discovery of refractory minerals [16], chondrule-like objects [17], and unexpectedly low abundances of presolar grains [18] and intact organic materials [19]. An even greater challege has been to locate and analyze samples of the interstellar dust stream targeted by the Stardust spacecraft [20]. Only a few submicrometer grains have been found so far and the critical analyses necessary to determine whether or not they are true insterstellar grains are still in the planning stages [21].

The analysis of Hayabusa asteroidal regolith samples poses its own challenges due to the limited amount of sample returned. Work on these samples is proceeding in a consortium fashion particle by particle. Despite this limitation, researchers have already confirmed that $\mathrm{S}$ type asteroids are likely parent bodies of ordinary chondrite meteorites commonly found on Earth [14]. Investigations of space weathering and radiation damage are another area of intense interest [22] as these are the first samples directly obtained from the surface of an asteroid.

Each of these collections yield unique types of information about the nature and origin of interplanetary dust particles and their source bodies, but none are free of collection bias. Direct samples of asteroids and comets are extremely valuable because they provide fresh materials with well-established geochemical context and strengthen connections between remote observations and sample analyses. But, for practical reasons the number of bodies that can be 

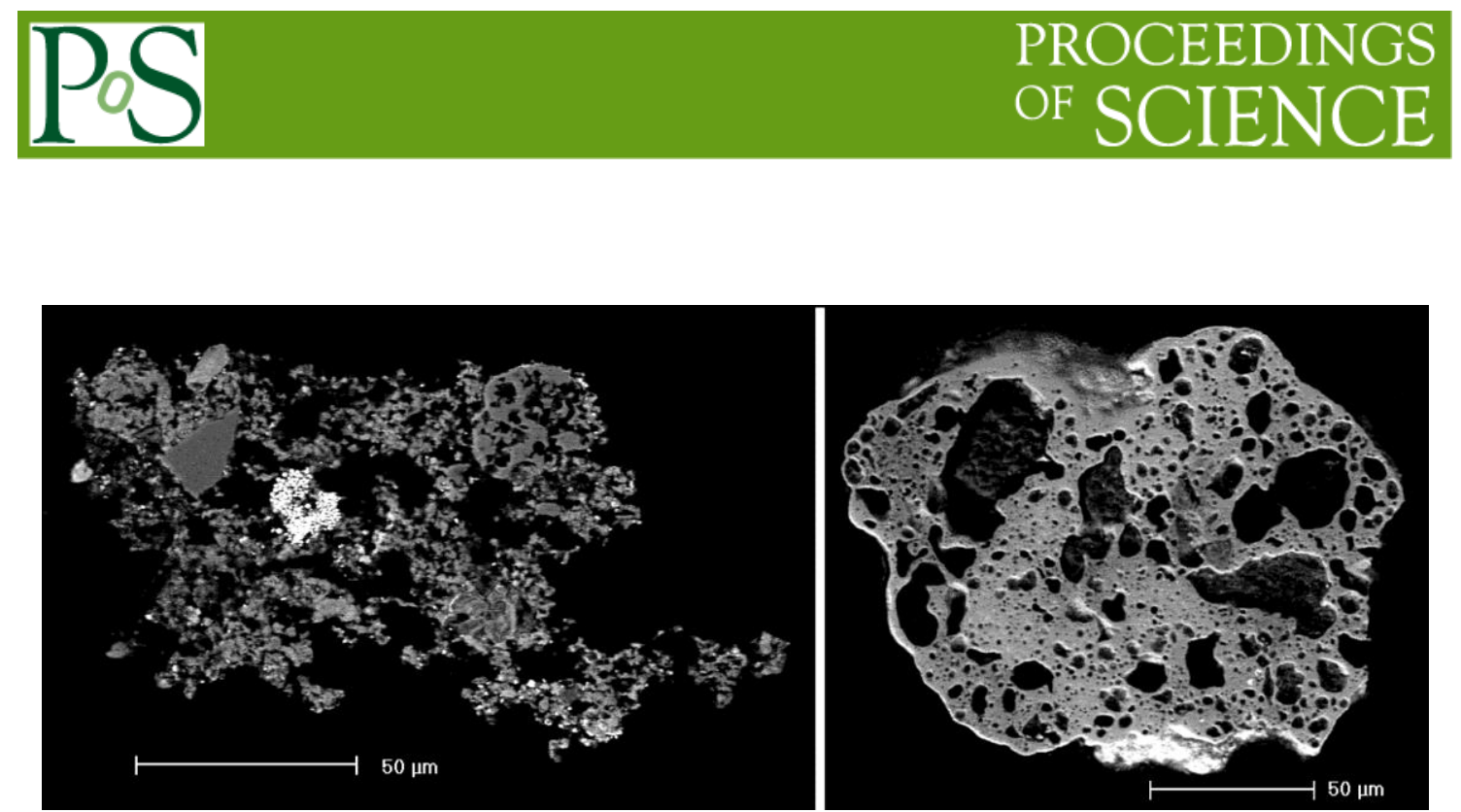

Fig. 1: (Left) Fine grained micrometeorite with a large pyroxene grain and framboidal magnetite. (Right) Scoriaceous micrometeorite. SEM backscattered electron images courtesy of Matt Genge.

sampled in this way is small compared to the diversity of potential targets. Terrestrial cosmic dust collections provide a broad sampling of dust-producing asteroids and short-period comets. However, high velocity particles (typically cometary) and many micrometeorites are altered or destroyed by atmospheric entry heating. Particles collected on or near the Earth surface are also susceptible to contamination and weathering. These shortcomings may be overcome by careful sample selection and by quantifying alteration effects related to terrestrial residence or sample collection.

\subsubsection{Micrometeorites}

Micrometeorites are $\sim 25-500 \mu \mathrm{m}$ extraterrestrial particles that are collected on the Earth surface. These particles represent the peak of the mass flux of extraterrestrial materials accreted by the Earth $(\sim 200 \mu \mathrm{m}[12])$ and therefore sample the dominant dust producing bodies in the Solar System in recent times. Micrometeorites were first identified in samples of deep sea sediments in the $19^{\text {th }}$ century as 'cosmic spherules,' consisting of rounded metal or silicate grains with magnetite on their surfaces [23, 24]. These textures are consistent with melting as a result of frictional heating during entry into the Earth's atmosphere. Recent expeditions to Antarctica and Greenland have collected thousands of micrometeorites, many of which survived atmospheric entry without melting [25-27]. The polar micrometeorites show diverse textures and compositions that largely overlap with those of primitive carbonaceous chondrite meteorites, although there are also differences.

Micrometeorites are classified according to both their mineralogy and texture and the extent of thermal alteration resulting from atmospheric entry heating. Heating effects vary from unmelted particles that preserve thermally sensitive phases such as phyllosilicates and organics to completely melted cosmic spherules. Micrometeorites that experienced partial melting are termed scoriaceous because they possess irregular vesicles that resemble the bubbly textures of dark volcanic rock (Fig. 1, [25]). Small $(<50 \mu \mathrm{m})$ particles generally show the lowest degrees of heating, but peak temperatures reached during atmospheric entry are also strong functions of 


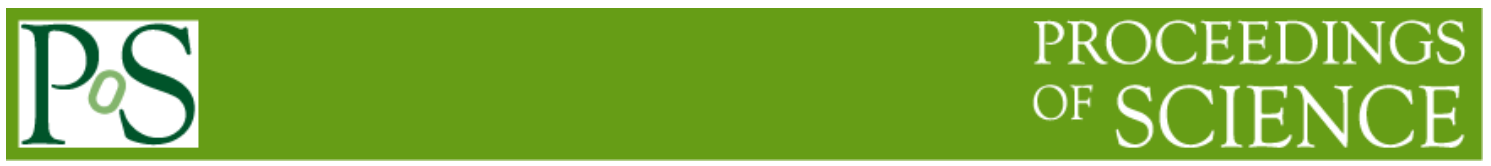

Earth-encounter velocity, angle of entry into the atmosphere and particle densities and compositions. Unmelted micrometeorites are broadly classified into coarse grained micrometeorites that are dominated by >several $\mu \mathrm{m}$-sized anhydrous crystalline silicates in glassy mesostasis and fine grained micrometeorites that are mostly sub- $\mu \mathrm{m}$ mineral grains [28].

Fine grained micrometeorites are typically composed of hydrated silicates or their thermal decomposition products, olivine, and pyroxene, with varying proportions of magnetite, metal, sulfides, and refractory minerals. These particles likely derive from parent bodies that were warm enough to melt ice and sustain liquid water in their interiors. This evidence of aqueous alteration is very common among primitive carbonaceous chondrite meteorites [29]. The mineralogy of fine grained micrometeorites is indeed similar to the matrix of type CM, CI, and CR chondrite meteorites [30]. Variations in the textures and mineralogy of these particles reflect differing extents of parent body aqueous alteration that cover the range of alteration recorded in meteorites. Rare micrometeorites are also found that contain refractory minerals that are probably fragments of refractory inclusions that occur in primitive meteorites.

Coarse grained micrometeorites are igneous objects that are mainly composed of crystalline olivine and pyroxene within a glassy mesostasis, metal and sulfides. Most coarse grained micrometeorites have mineralogy similar to chondritic meteorites, although rare cases of achondrite-like micrometeorites and single crystals are also found. The textures and mineralogy of coarse grained micrometeorites are often similar to chondrules. It is not certain what fraction of micrometeorites may be derived from chondrules, but chondrules are common components of chondritic meteorites, so their presence among the micrometeorite population is expected. Some coarse grained micrometeorites are decorated with fine grained chondritic material that indicates coarse- and fine-grained micrometeorites may derive from the same parent bodies in some cases.

Overall, the wide range of micrometeorite textures and compositions are consistent with known classes of chondritic meteorites. However, fragile materials would be unlikely to survive hundreds to thousands of years trapped in ice and the recovery process. A new collection of micrometeorites has recently been obtained from freshly fallen snow that contains small, fragile 'ultracarbonaceous' particles with properties distinct from previously collected micrometeorites and carbonaceous chondrite meteorites [27, 31]. The particles are first distinguished by their very high $\mathrm{C}$ abundances ( $\sim 50 \mathrm{wt} . \%$ or more) that greatly exceed the most $\mathrm{C}$-rich meteorites (a few wt. \%). Moreover, their mineralogy is very similar to the anhydrous chondritic porous interplanetary dust particles discussed in the following section. Based on their high $\mathrm{C}$ content and anhydrous mineralogy, a cometary origin for these particles is proposed, but this remains uncertain.

\subsubsection{Stratospheric Interplanetary Dust Particles (IDPs)}

Interplanetary dust was first collected in the upper stratosphere by high altitude (>35 km) balloon flights [32]. At this altitude most of the atmospheric dust particles $>10 \mu \mathrm{m}$ were found 

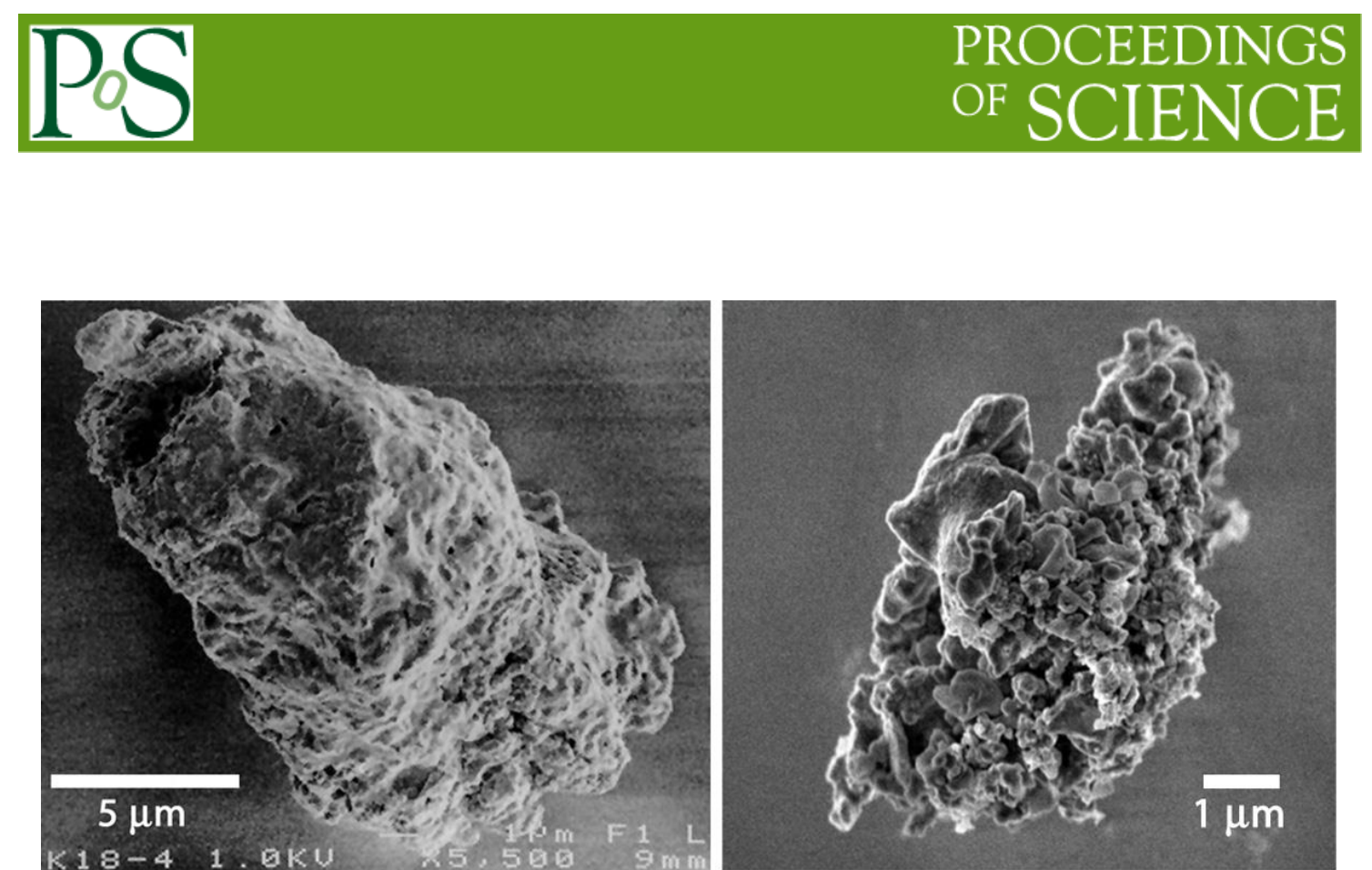

Fig. 2: (Left) SEM image of a CS hydrated IDP of probable asteroidal origin. (Right) SEM image of a CP anhydrous IDP of probable cometary origin. These particles can be viewed as samples of planetary materials (CS-IDPs) and nebular/interstellar materials (CP-IDPs).

to have extraterrestrial origins. Following this success, NASA established routine collections of cosmic dust by high altitude $(18-20 \mathrm{~km})$ flights of U2, ER-2, and WB-57 aircraft [24, 33]. Particles are collected by inertial impact onto flat plates coated with silicone oil to prevent bouncing or oil-free polyurethane foam to trap impacting particles [34].

Particles are investigated for their textures and bulk major element abundances by scanning electron microscopy energy dispersive X-ray analysis (SEM-EDX) after oil has been removed with an organic solvent (hexane). The compositions of the particles reflect both terrestrial and extraterrestrial origins. Common terrestrial contaminants include volcanic dust, solid rocket exhaust particulates (aluminum oxide spherules), industrial pollutants, paint chips, metal grains, skin flakes, and other materials of uncertain origin. Excluding rocket exhaust and volcanic dust, whose abundances vary considerably, approximately half of the collected particles have bulk element abundances that are within a factor of 3 of primitive CI chondrite meteorites [35]. Several independent proofs of extraterrestrial origins of such 'chondritic' particles have been established over time, including the observation of solar flare tracks, implanted solar noble gas, and non-terrestrial $\mathrm{H}, \mathrm{C}, \mathrm{N}$, and $\mathrm{O}$ isotopic abundances [35-40].

Stratospherically collected interplanetary dust particles (IDPs) have characteristics that generally set them apart from the larger micrometeorites and meteorites collected on the Earth surface. IDPs occur in several broad compositional and textural classes: (1) chondritic, smooth (CS) and compact particles that are mainly composed of hydrated silicates (2) chondritic, porous (CP) particles that are dominated by anhydrous silicates and (3) large (1-10 $\mu \mathrm{m})$ single mineral grains such as $\mathrm{Fe}$, Ni sulfides and mafic silicates that may have small amounts of fine-grained chondritic material adhering to them [41].

Chondritic-smooth IDPs (Fig. 2) are low porosity objects whose mineralogy is dominated by aqueous alteration products such as $\mathrm{Mg}$-rich phyllosilicates and $\mathrm{Mg}$-Fe carbonate minerals. 

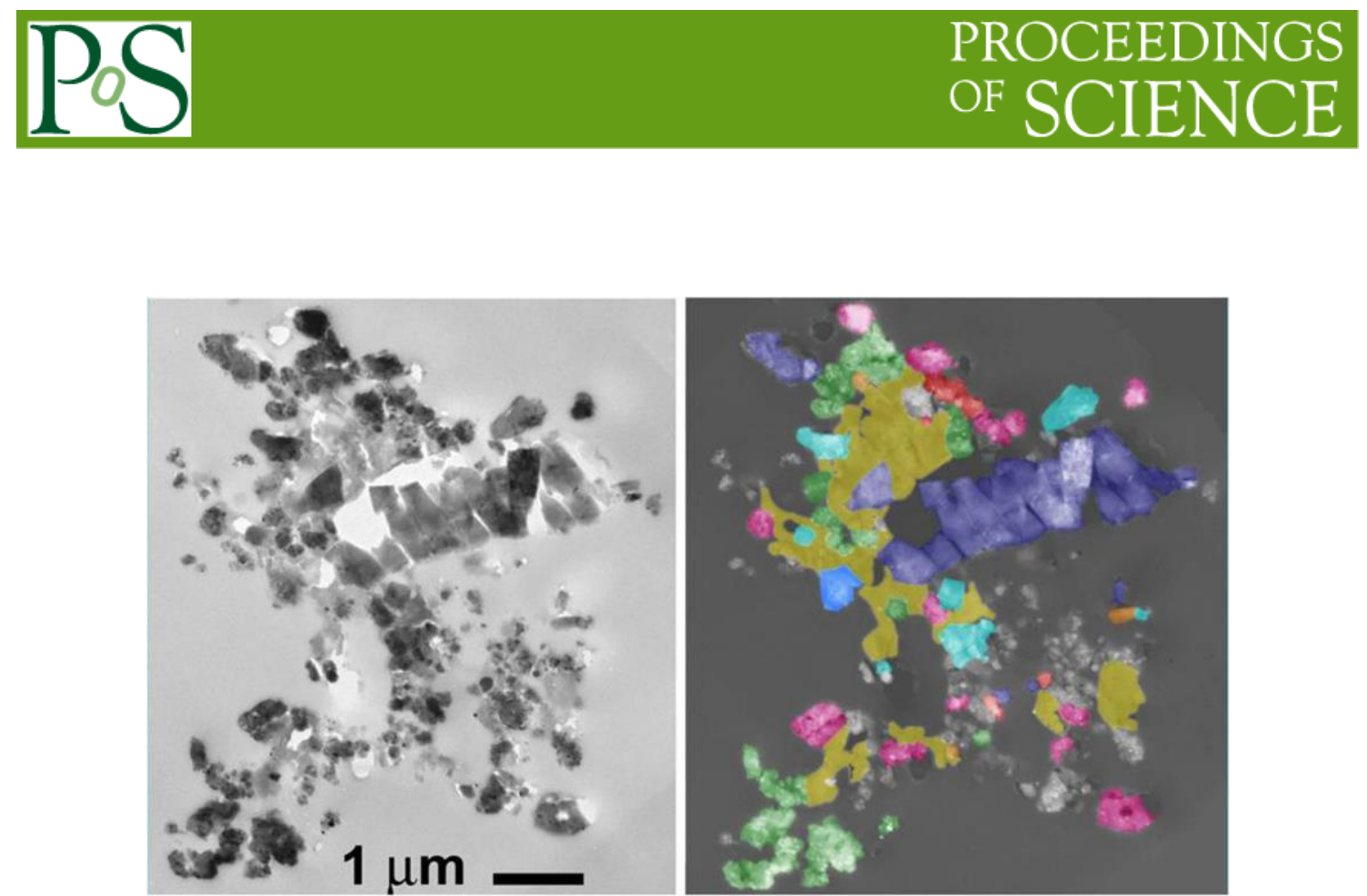

Fig. 3: (Left) Bright field TEM image of a 70 nm-thick section of a CP IDP. (Right) False color mineral map of the IDP. Enstatite $=$ dark blue, forsterite $=$ light blue, iron sulfide $=$ red, $\mathrm{Ca}, \mathrm{Al}-$ rich glass $=$ orange, equilibrated aggregates $=$ purple, amorphous silicates $($ GEMS $)=$ green, carbonaceous material $=$ yellow .

CS-IDP mineralogy shows broad similarities to primitive carbonaceous chondrites and a few particles have been directly linked to the parent bodies of CM and CI chondrites based on their compositions and presence of specific minerals [42-46]. However, some CS-IDPs have apparently experienced only localized aqueous processing, as evidenced by greater compositional heterogeneity compared with CM and CI chondrites [42]. CS-IDPs also typically have carbon contents that greatly exceed that of known meteorite groups [47-49]. The Fe-Ni sulfide minerals in CS-IDPs tend to be more Ni-rich than those in CP-IDPs, presumably reflecting the oxidizing nature of the aqueous fluids [50].

Chondritic-porous IDPs are highly fragile objects (Fig. 2). Because of their low strength, they often break into hundreds of fragments upon impacting cosmic dust collector surfaces or during sample preparation. Transmission electron microscope studies reveal that CP-IDPs are assemblages of sub- $\mu \mathrm{m} \mathrm{Mg-rich} \mathrm{olivine} \mathrm{and} \mathrm{pyroxene} \mathrm{grains,} \mathrm{Fe-Ni} \mathrm{sulfides,} \mathrm{and} \mathrm{amorphous}$ silicates all bound together by abundant organic-rich carbonaceous material (Fig. 3). The proportions of these phases are highly variable from particle to particle, and any one phase may dominate individual CP-IDPs. On average, the $\mathrm{C}$ abundance of CP-IDPs is $2-3 \mathrm{x}$ that of the Crich CI chondrite meteorites [51].

Crystalline silicates in CP-IDPs are dominated by enstatite $\left(\mathrm{MgSiO}_{3}\right)$ and forsterite $\left(\mathrm{Mg}_{2} \mathrm{SiO}_{4}\right)$, with minor phases including diopside $\left(\mathrm{CaMgSi}_{2} \mathrm{O}_{6}\right)$ and anorthite $\left(\mathrm{CaAl}_{2} \mathrm{Si}_{2} \mathrm{O}_{8}\right)$ [52]. The microstructure and compositions of enstatite and forsterite in CP-IDPs are consistent with condensation from a high temperature gas [53]. Equilibrium condensation calculations show that these silicates begin to condense from a gas of solar composition over a relatively narrow temperature interval ( $1380-1310 \mathrm{~K}$ at $10^{-4}$ bar total pressure [54]). Many enstatite grains exhibit unusual crystal morphologies such as whisker and platelet forms that, along with growth 


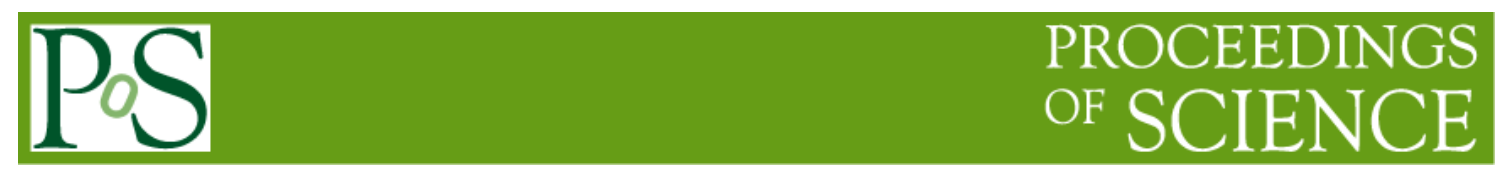

defects, are characteristic of vapor phase growth [55]. Crystalline silicates typically occur as single crystals ranging in size from $0.1 \mu \mathrm{m}$ to several $\mu \mathrm{m}$, but polycrystalline grains are also common and are referred to as "equilibrated aggregates" [56, 57].

Equilibrated aggregates (EAs) are 0.1-2.0 $\mu \mathrm{m}$ sized irregularly-shaped, polycrystalline grains (Fig. 4). Their abundance in individual IDPs is highly variable from a few vol. \% to nearly the entire volume of an IDP. The EAs consist of crystalline grains with a simple mineralogy dominated by $\mathrm{Mg}$-rich

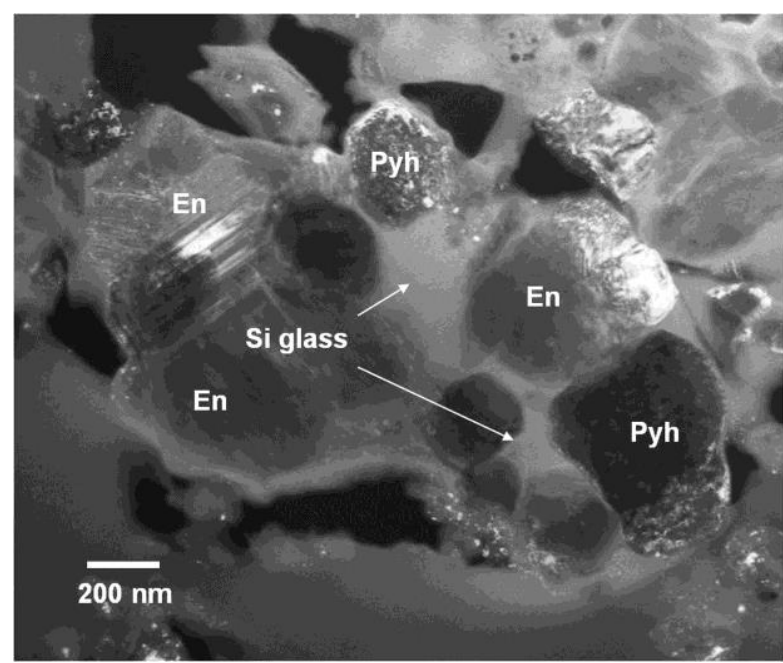

Fig. 4: TEM darkfield image of an equilibrated aggregate (EA) in a CP IDP. En = enstatite, Pyh=pyrrhotite.

enstatite and forsterite, pyrrhotite, $\mathrm{Mg}$ - $\mathrm{Al}$, Si-rich mesostasis, and minor diopside. The constituent grains in EAs are euhedral and range from 50-200 nm in size, but are typically 100 $\mathrm{nm}$ in size and frequently show equilibrium grain boundaries. The mineralogy and irregular morphologies of EAs show they were never molten, and their textures are consistent with formation by subsolidus annealing of amorphous precursors [57, 58].

Amorphous silicates are an abundant constituent of CP-IDPs, accounting for as much as half the mass in some particles. The most common type of amorphous silicates is termed GEMS (glass with embedded metal and sulfides; [59, 60]). GEMS grains are $\sim 50-500 \mathrm{~nm}$ rounded or irregular amorphous $\mathrm{Mg}$-Si-Al-Fe silicate grains that contain numerous 10 to $50 \mathrm{~nm}$-sized FeNi metal and Fe-Ni sulfides. The origins of GEMS grains are discussed in more detail below.

Iron-nickel sulfides are the other major crystalline constituent of CP-IDPs. Their mineralogy is dominated by low-Ni pyrrhotites $\left[\mathrm{Fe}_{(1-\mathrm{X})} \mathrm{S}\right]$ with a wide range of grain sizes [50, 61]. Pyrrhotites occur as isolated single crystals up to several $\mu \mathrm{m}$ in size as well as 10-50 nmsized grains associated with amorphous silicate grains. A cubic Fe-sulfide $\left(\mathrm{Fe}_{3} \mathrm{~S}_{4}\right)$ has also been reported in CP-IDPs that may form through condensation from the gas phase [61]. Zinc- and Nirich sulfides are also reported as trace phases in CP-IDPs [50, 62, 63]. Most Fe-Ni sulfides are believed to form at relatively low temperatures through the sulfidization of pre-existing FeNi metal in the early nebula [64]. Tiny sulfide grains decorate the surfaces of some amorphous silicate grains in CP-IDPs and thus represent a late addition of material during their formation [52].

CP-IDPs are usually fully anhydrous samples that appear to have escaped significant postaccretional alteration. However, rare "hybrid" IDPs occur that have typical CP-IDP mineralogy but also contain small amounts of material that partly reacted with aqueous fluids to form hydrated minerals typical of CS-IDPs [65]. Experiments have shown that the anhydrous 


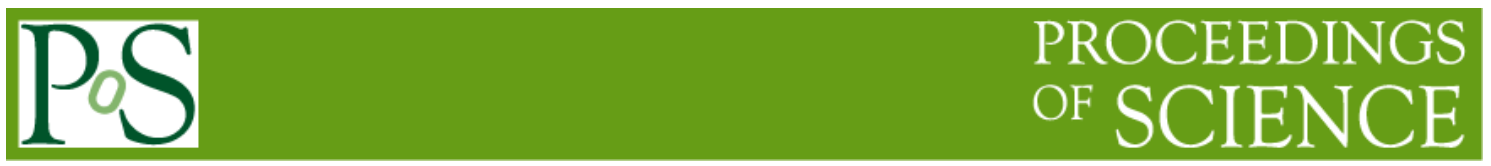

mineralogy of CP-IDPs is highly reactive in the presence of aqueous fluids [65]. The amorphous silicates are especially prone to aqueous alteration and can be hydrated in as little as a day at room temperature.

\subsubsection{Constraints on IDP source bodies}

There is considerable interest in determining the sources of IDPs because many clearly originate from primitive bodies that are different from those sampled by meteorites. Multiple lines of evidence suggest that CP-IDPs derive from short-period comets. First, their fine grained and fragile structure is consistent with properties of comets as inferred from the low strength of cometary meteors and the abundance of sub- $\mu \mathrm{m}$ grains observed in cometary comae $[66,67]$. Second, CP-IDPs have C abundances that are more similar to comets than meteorites [51]. Third, CP-IDPs have high abundances of presolar grains and molecular cloud material [68-70]. Finally, CP-IDPs have experienced minimal parent body alteration, as shown by highly unequilibrated and anhydrous mineralogy. Although comets are rich in water ice, it has not been considered possible for them to host liquid water, so cometary solids are expected to be anhydrous. The hybrid IDPs are an interesting counterpoint to this argument because they contain traces of materials that formed under the action of water. Recent observations of low temperature sulfide minerals in Stardust comet 81P/Wild 2 returned samples suggest that limited aqueous activity occurred on comet Wild 2 [71]. On the other hand, CS-IDPs originate from parent bodies that underwent extensive aqueous alteration, conditions that are known to have occurred in some asteroids, but are considered unlikely to occur in comets. Accordingly, CSIDPs are usually ascribed asteroidal origins [72, 73]. The high $\mathrm{C}$ abundances of CS-IDPs are a puzzle if this is the case because such $\mathrm{C}$-rich materials are rare among the meteorite collection. It is possible that CS-IDPs derive from primitive C-rich asteroids that are not sampled by meteorites. Thus, the compositional differences between CS-IDPs and meteorites may simply reflect the fact that interplanetary dust may escape any portion of the asteroid belt due to Poynting-Robertson drag, whereas larger bodies are more dynamically confined.

The inferences of IDP parent bodies based on composition and structure are plausible but are uncertain, given our limited knowledge of the properties of comets and the limited sampling of asteroids. The strongest direct evidence constraining the sources of IDPs is based on their atmospheric entry velocities. Dust particles from comets have higher orbital eccentricities and inclinations compared with asteroidal dust. This is manifested as a significantly higher Earthencounter velocity for cometary dust than asteroidal dust. After thousands of years in space, these differences are lessened, but the general trend is maintained. The Earth-encounter velocity differences result in differing degrees of heating during atmospheric entry. Peak temperatures of IDPs inferred by the stepwise thermal release of solar wind He show systematically higher values for CP-IDPs, and thus higher estimated atmospheric entry velocities $(>18 \mathrm{~km} / \mathrm{s})$ than CSIDPs $(<14 \mathrm{~km} / \mathrm{s}$; [74]). These findings support the association of CP-IDPs with short period comets and CS-IDPs with asteroids. However, there is enough overlap between the groups that 


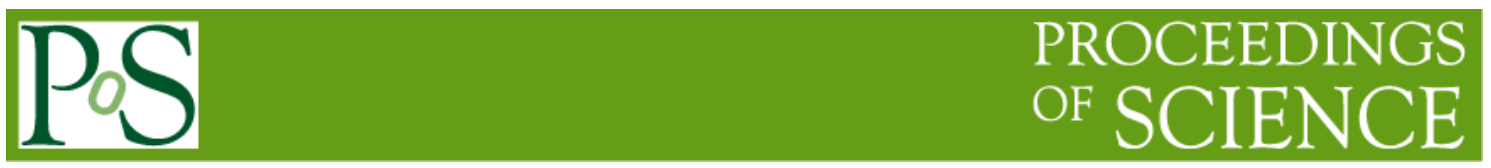

the possibility remains that some CP-IDPs derive from asteroids and some CS-IDPs are cometary.

CP and CS-IDPs are collected at similar rates in the stratosphere, but this is unlikely to reflect the proportions of cometary and asteroidal dust in the inner Solar System. On one hand, CP-IDPs are over-represented in the collection because their lower density results in slower settling rates in the atmosphere and thus have higher atmospheric concentrations. On the other hand, interplanetary dust originating from asteroids tends to have lower orbital eccentricity than cometary dust, resulting in a gravitational focusing effect that favors the capture of asteroidal over cometary dust [75]. A dedicated space-based dust collector or detector capable of measuring particle trajectories is probably required to accurately measure the proportions of cometary and asteroidal dust in the inner Solar System.

\subsubsection{Nebular and interstellar components within IDPs}

The question of where and how the components within IDPs formed is even more important than the question of what the source bodies of IDPs are. A typical $10 \mu \mathrm{m}$ CP-IDP may contain over $10^{5}$ distinct mineral and organic grains. Deciphering the origins and histories of these individual components is a great challenge and is an area of active research. Any given sub-grain may in principal derive from one of the following sources: (1) the interstellar medium or stellar ejecta (2) the solar nebula (3) a parent body separate from that of the IDP source (4) the IDP parent body itself. Types of materials most likely to form or be altered in parent body settings include hydrated minerals or byproducts of hydrothermal alteration, products of thermal alteration or shock (from impacts) and organic materials that may form from aqueous solutions or thermal processes. CS-IDP parent bodies have undergone extensive hydrothermal processing and it is likely that these samples are complex mixtures of materials having nebular and interstellar origins and secondary alteration products. On the other hand, CP-IDPs show very little evidence of parent body hydrothermal processing that has affected the mineralogy of even the most primitive meteorites [76]. Consequently, CP-IDPs are regarded as being essentially pristine aggregates of nebular and interstellar materials.

If CP-IDPs have escaped parent body processing, the question of where CP-IDP components formed simplifies into Solar System versus interstellar (presolar) origins. Detailed mineralogical studies by TEM yield critical constraints on formation processes, but cannot discern where the materials formed. At present, isotopic measurements provide the only definitive way of discerning interstellar from Solar System origins. The Solar System starting materials consisted of stardust grains and interstellar molecular cloud materials having a wide range of isotopic compositions, in some cases orders of magnitude variations in a single element [77]. By contrast, Solar System materials are well homogenized and typically display percentlevel isotopic variations in most elements, arising from kinetic effects during physical and chemical processes. Coordinated mineralogical, chemical, and isotopic studies are needed to elucidate both the formation processes and where the components originated. 


\subsection{Ancient interstellar dust}

It is a remarkable fact that stardust grains that predate the formation of the Solar System have survived for billions of years in primitive Solar System bodies. These grains are direct samples of remote astrophysical environments that are difficult or impossible to observe by traditional astronomy. The laboratory study of stardust has had an enormous impact on our understanding of stellar nucleosynthesis, galactic chemical evolution, grain condensation processes in stellar atmospheres, and the lifecycle of dust in the Galaxy. Here we present a brief overview of the properties of presolar grains that relate to the nature of the Solar System starting materials. Detailed reviews of the isotopic properties of presolar grains, relevant nucleosynthetic interpretations, and historical perspectives can be found in [78-81].

The discovery of presolar grains in meteorites followed a painstaking search for the carriers of isotopically anomalous noble gas components in meteorite residues. The carrier phases were gradually concentrated by trial and error, applying strong acid and other chemical treatments and physical separations of meteorite materials. This strategy gradually teased out the rare (ppm abundance) presolar dust grains from the host meteorite in a process that was, as team leader Ed Anders put it, like 'burning down the haystack to find the needle.' The first presolar phases identified were diamond, $\mathrm{SiC}$, and graphite [82-84]. The $\mathrm{SiC}$ and graphite grains were shown to have stellar origins on the basis of highly anomalous (non-solar) isotopic compositions. SiC and graphite grains are large enough $(\sim 0.1-40 \mu \mathrm{m})$ that it is possible to measure their major and minor element isotopic compositions in individual grains by secondary ion mass spectrometry (SIMS). Their mineralogical identifications were established by electron diffraction and high-resolution imaging by transmission electron microscopy.

Carbon and nitrogen isotopic ratios measured in presolar SiC grains span four orders of magnitude (Fig. 5). The enormous ranges in isotopic compositions are the result of nucleosynthetic processes in the stars that produced these grains. Dust from asymptotic giant branch (AGB) stars is formed from the products of $\mathrm{H}$ and $\mathrm{He}$ burning and $s$-process nucleosynthesis, while nova and supernova dust is imparted with the products of later stages of nuclear burning, explosive nucleosynthesis, and presumably the $r$-process. The isotopic ratios of material inherited by the dust grains are also strongly dependent on the masses, chemical compositions and evolutionary stages of the parent stars at the time that each grain was formed. Therefore, it is possible to infer the properties of the parent star from the isotopic composition of a given dust grain.

$\mathrm{SiC}$ is the most thoroughly studied presolar grain type because of its relatively large size and high abundance of trace elements. The development of automated isotopic imaging systems has made it possible to amass an impressively deep survey of presolar SiC, with isotopic data from over 40,000 grains published in an online database [85]. These data are classified into isotopic subgroups such as mainstream, $\mathrm{A}+\mathrm{B}, \mathrm{X}, \mathrm{Y}$, and $\mathrm{Z}$ grains that are ascribed to distinct stellar sources. The majority of presolar SiC grains ( $\sim 90 \%)$, termed 'mainstream,' originated from low-mass AGB stars of near solar metallicity, while $\mathrm{Y}$ and $\mathrm{Z}$ grains (a few \%) are thought 


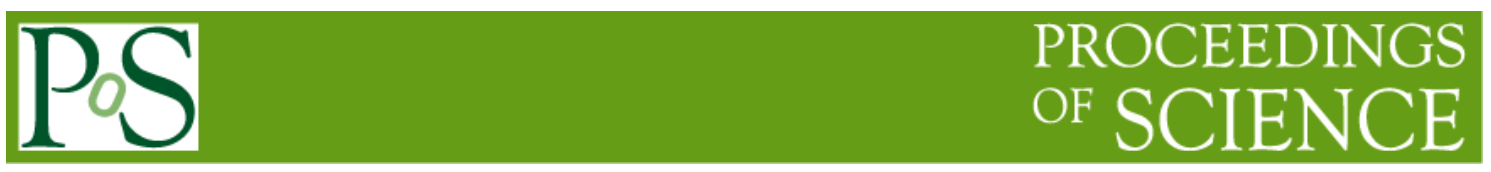

to derive from low-metallicity AGB stars. $\mathrm{X}$ grains $(\sim 1 \%)$ have been shown to derive from supernovae on the basis of extreme isotopic enrichments in ${ }^{44} \mathrm{Ca}$ that is attributed to the decay of ${ }^{44} \mathrm{Ti}\left(\tau_{1 / 2}=\right.$ 60 years) [86]. The only known source of ${ }^{44} \mathrm{Ti}$ is in the deep interior layers of type II core-collapse supernovae [87]. The low ${ }^{12} \mathrm{C} /{ }^{13} \mathrm{C}$ isotope ratios of type $\mathrm{A}+\mathrm{B}$ grains (few \%) are not easily reconciled with models of AGB stars. J-type carbon stars are leading candidates for their stellar sources. Some presolar $\mathrm{SiC}$ grains appear to be rounded or fractured, but most grains are exceptionally well preserved ([88], Fig. 6), reflecting a range of alteration histories and/or residence times in the interstellar medium.

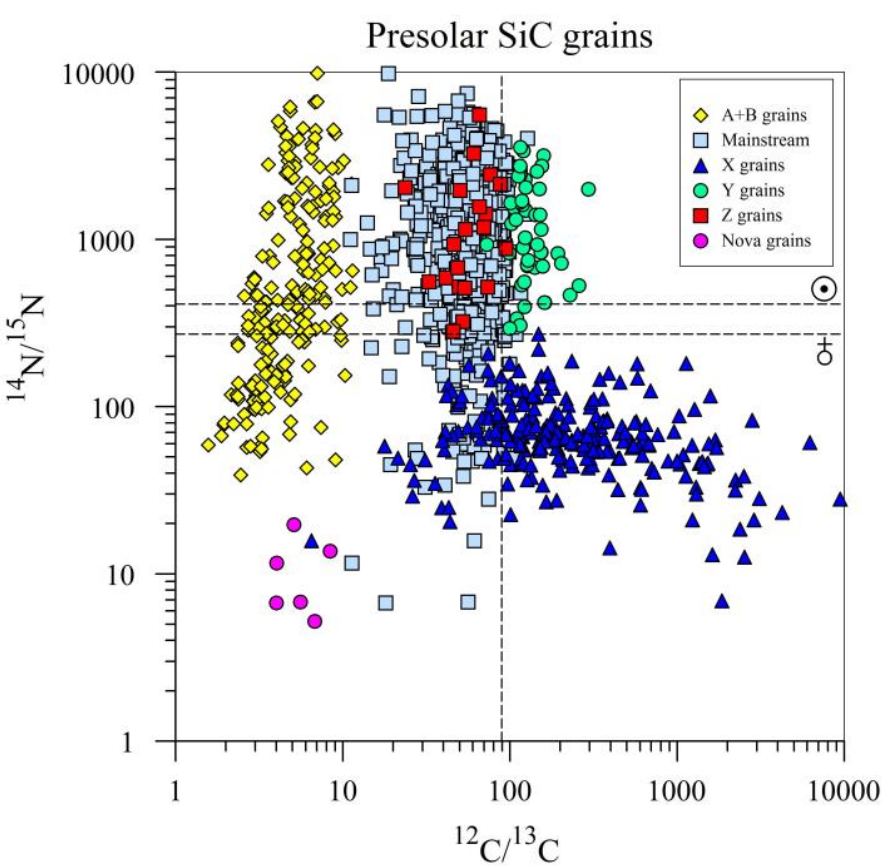

Fig. 5: $\mathrm{C}$ and $\mathrm{N}$ isotopic compositions of individual $\mathrm{SiC}$ grains extracted from primtive meteorites. The symbols correspond to isotopic subtypes that relate to distinct stellar origins. The Solar System ${ }^{12} \mathrm{C} /{ }^{13} \mathrm{C}$ isotope ratio and the terrestrial and solar ${ }^{14} \mathrm{~N} /{ }^{15} \mathrm{~N}$ isotope ratios are shown for reference as dashed lines. Data from [85] \& refs. therein.

Presolar graphite grains show similar ranges of isotopic signatures, indicating origins from carbon stars, novae, and supernovae. The proportion of graphite grains deriving from supernovae is thought to be much higher than is the case for $\mathrm{SiC}$, though the proportions vary according to the physical properties of the graphite grains. In the lowest density graphite (1.6$\left.1.7 \mathrm{~g} / \mathrm{cm}^{3}\right) 76 \%$ of grains are estimated to derive from supernovae, while this drops to just $1 \%$ in the highest density fraction $\left(2.1-2.2 \mathrm{~g} / \mathrm{cm}^{3}\right.$; [89]). Presolar graphite grains are especially interesting because they contain high numbers of tiny refractory carbide inclusions that in some cases appear to have served as seed nuclei for the graphite growth ([90], Fig 6). These are mainly $\mathrm{Ti}$ carbide grains having high concentrations of $\mathrm{Mo}, \mathrm{Ru}$, and $\mathrm{Zr}$ in solid solution, reflecting the enhancement of these s-process elements freshly formed in the host AGB stars $[91,92]$. It is noteworthy that isotopically anomalous polycyclic aromatic hydrocarbons (PAHs) have been measured in presolar graphite grains, effectively probing the chemical compositions of carbon star atmospheres [93].

The nanodiamonds have less certain origins than the $\mathrm{SiC}$ and graphite grains because their mean size is so small $(2.8 \mathrm{~nm})$ that it is still not possible to accurately measure the isotopic composition of a single nanodiamond [94]. A presolar origin is ascribed to some fraction of nanodiamonds because they are carriers of isotopically anomalous Xe that is enriched in both 


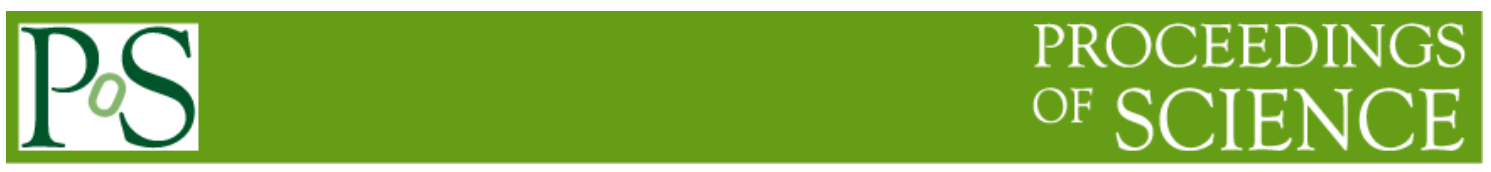

the heavy and light isotopes (Xe $\mathrm{HL}$ ), corresponding to the $p$ and $r$-processes in stars [83]. However, on average only one of every $\sim 10^{6}$ nanodiamonds contains a single $\mathrm{Xe}$ atom. Furthermore, their ${ }^{13} \mathrm{C} /{ }^{12} \mathrm{C}$ and ${ }^{15} \mathrm{~N} /{ }^{14} \mathrm{~N}$ isotopic ratios are, on average, within error of the composition of terrestrial materials and of the Sun, respectively [95]. It may be that most of the nanodiamonds formed within the Solar System and only rare nanodiamonds have stellar origins.

Following

these discoveries, there was great interest in finding presolar oxides and silicates, as these probe different evolutionary stages of stars from the C-rich presolar grains and because of the desire to more closely link laboratory studies of stardust
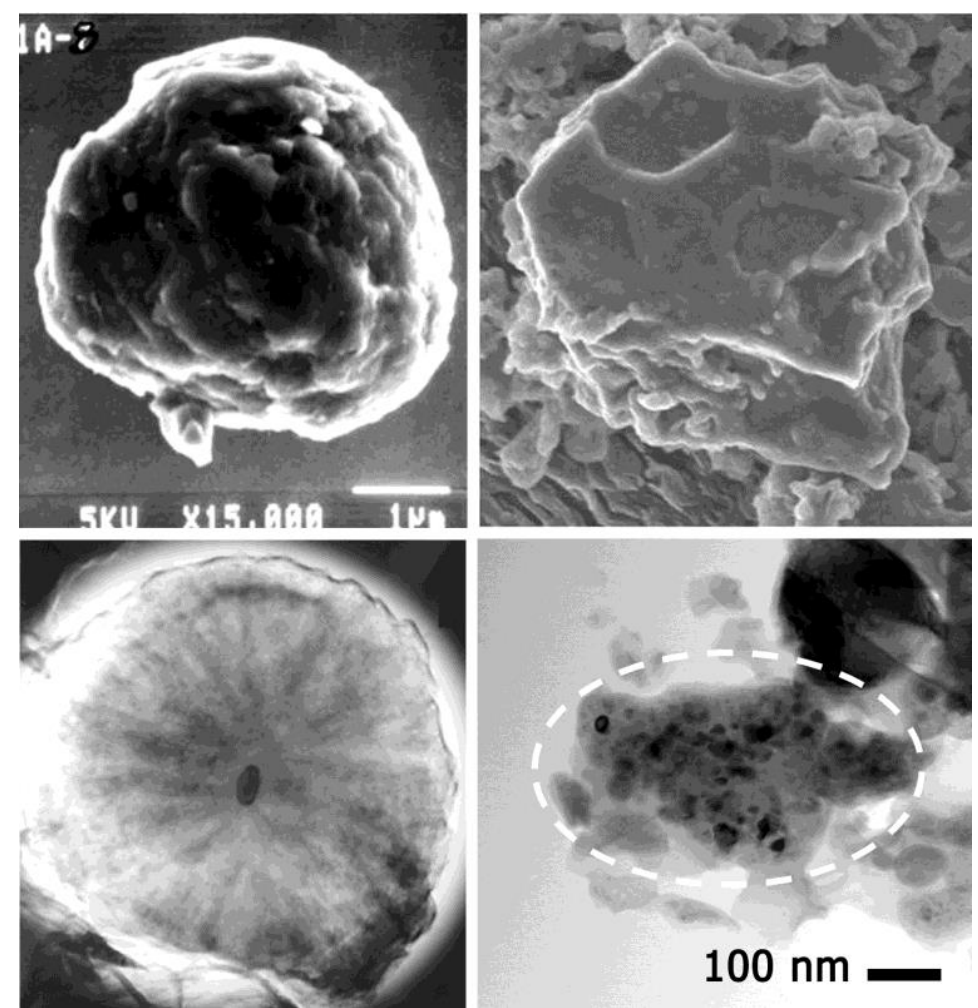

Fig. 6: (Top L) SEM image of a presolar graphite grain. (Top R) SEM image of a presolar SiC grain showing primary growth features [88] (Bottom L) Bright field TEM image of a $70 \mathrm{~nm}-$ thick section of $\mathrm{a} \sim 1 \mu \mathrm{m}$ presolar graphite grain having a large TiC internal grain. Image courtesy of Thomas Bernatowicz. (Bottom R) Bright field TEM image of a presolar GEMS grain. Dark spots are metal and sulfide grains.

with astronomical observations. The search for presolar oxides and silicates is complicated by the huge background of Solar System materials of similar mineralogy that make up the host meteorites and cometary dust. Presolar oxides were identified in acid residues of primitive meteorites, where roughly $1 \%$ of the oxides measured (depending on the meteorite) turned out to be a presolar grain $[96,97]$. This work was made much more efficient by the development of automated isotopic imaging systems, enabling hundreds of presolar oxide grains to be found [98]. Although presolar silicates are the most abundant presolar grain phase, they were among the last to be found due to their small size ( $200 \mathrm{~nm})$. The discovery of silicate stardust in IDPs [40] and meteorites [99] was made possible by the high spatial resolution $(<100 \mathrm{~nm})$ and high transmission of the newly introduced NanoSIMS ion microprobe, and later by a new 'SCAPS' imaging system for the IMS 1270 ion probe [100]. Similar to presolar SiC and graphite grains, presolar oxides and silicates show a range of isotopic ratios that indicate origins in red giant stars, novae, and supernovae. 


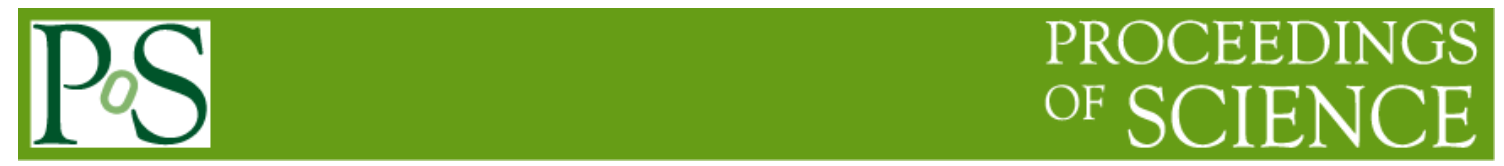

The laboratory study of presolar silicates enables direct tests of interstellar grain models that have been based solely on spectroscopic studies for decades. Silicates are less refractory and more susceptible to alteration or destruction by thermal, radiation, shock, and aqueous processes compared with other types of presolar grains, and thus offer unique insight into the histories of interstellar dust and early Solar System processes. Detailed mineralogical studies of presolar silicates are challenging due to their small size, but two successful strategies have been employed: isotopic imaging to first identify presolar silicates followed by focused ion beam (FIB) lift-out for TEM study and mineralogical mapping by TEM followed by isotopic imaging. Over time, a picture of silicate stardust mineralogy has emerged that appears to defy simple models of interstellar dust compositions.

Spectroscopic observations of material ejected from evolved stars, interstellar molecular clouds, and young stellar systems have established a consistent picture of the formation and evolution of silicate dust in the Galaxy. Astronomical observations of O-rich AGB stars indicate the majority of the circumstellar silicate dust is amorphous with olivine-like, Fe-bearing chemical compositions. The proportion of crystalline silicate grains is estimated to be on average $10 \%$ of the silicate mass, with most of the grains being pyroxene and extremely $\mathrm{Mg}$ rich [101]. The silicate dust in the interstellar medium (ISM) is also mostly amorphous with Febearing olivine-like compositions. However, the proportion of crystalline silicates in the ISM is constrained to be less than $1 \%$ of the silicate mass, roughly 10 times lower than the amount produced by AGB stars [102]. This is attributed to amorphization in the ISM by processes such as ion irradiation, grain collisions, and supernova shock waves. The compositions and abundances of dust created during supernova explosions are not well constrained by astronomical observations. However, dust emission features have been fit to $\mathrm{Mg}$-rich amorphous silicates with both olivine- and enstatite-like compositions [103]. The characteristics of silicate dust produced in nova outbursts are even more uncertain, but similar to interstellar and circumstellar grains, most of the silicates are amorphous [104].

The detailed chemical compositions and crystal structures of $\sim 40$ presolar silicates have been determined by TEM analysis. The majority of these grains have origins in AGB stars, but analyses of rare nova and supernova grains have also been achieved. Silicates are more mineralogically and chemically diverse than other presolar phases. The presolar silicate mineralogies include Mg-rich crystalline olivine and pyroxene, partially crystalline grains, and non-stoichiometric amorphous silicates. Some of the presolar amorphous silicates are classified as GEMS grains (Fig. 6) while others are not associated with metal or sulfide grains. The crystalline silicate grains show a range of Fe-contents and the amorphous grains have a wide range of chemical compositions that are often spatially heterogeneous. Aggregates of presolar silicates [105] and silicate/oxide assemblages have also been observed.

The majority of presolar silicate grains ( $70 \%$ on average) are amorphous with nonstoichiometric chemical compositions. This is generally consistent with observations of silicate dust around evolved stars. However, the proportion of crystalline silicates is much higher than 
expected from observations of silicate dust in the ISM, indicating that some grains were annealed or that the amorphous grains are more susceptible to destruction by alteration processes in the solar nebula. While astronomical spectra indicate the majority of crystalline silicates have the composition of pyroxene, laboratory analysis of presolar silicates reveals crystalline olivine grains are approximately twice as abundant as pyroxene. These olivine grains also have substantial $\mathrm{Fe}$-contents while the pyroxene grains have no detectible Fe.

In general, the silicate grains have higher Fe-contents than predicted from equilibrium condensation theory. The $\mathrm{Fe}$ in these grains could have been acquired during condensation if it occurred under non-thermodynamic equilibrium conditions or if the grains formed at lower temperatures [106]. This scenario is probable for presolar silicates identified in pristine CP-IDPs or in non-stoichiometric grains with a uniform distribution of Fe. Alternatively, the Fe could have resulted from secondary alteration on the parent body. This is likely for grains in which the $\mathrm{Fe}$ is concentrated in the outer portions and for grains identified in meteorites that have experienced more thermal alteration.

Further evidence for secondary alteration has been observed. A presolar equilibrated aggregate identified in an IDP likely formed by annealing of amorphous precursors [107]. The unique perovskite-like crystal structure of a presolar $\mathrm{MgSiO}_{3}$ grain most likely resulted from shock processing in the ISM or from the parent star [108]. There is also evidence for radiation processing in some presolar silicates. One stoichiometric Mg-rich enstatite supernova grain is likely to have originally condensed as a crystal under equilibrium conditions but was later rendered amorphous either in the vicinity of the supernova remnant or at a later stage [109]. A similar scenario is likely for a presolar enstatite grain having a nanocrystalline core and amorphous shell [110].

Condensation theory predicts that silicate growth can only proceed on nm-sized oxide seed nuclei [111]. While sub-grains (mostly carbides) have been observed in abundance in presolar graphite and $\mathrm{SiC}$ grains, oxides are generally absent within presolar silicate grains. However, there has been a recent observation of a presolar crystalline spinel grain surrounded by an amorphous silicate mantle of heterogeneous nonstoichiometric composition [112]. The spinel core likely condensed under equilibrium conditions while the silicate material formed at lower temperature under non-equilibrium conditions. The structure of this grain attests to the rapidly changing chemical and physical

\begin{tabular}{|lll|}
\hline Phase & Tc $(\mathrm{K})$ & Reference \\
\hline Corundum & 1633 & $96-98$ \\
Hibonite & 1562 & 113 \\
Perovskite & 1537 & \\
Melilite & 1472 & \\
Spinel & 1351 & 114,115 \\
Plagioclase & 1320 & \\
Rankinite & 1318 & \\
Fassaite & 1317 & \\
Forsterite & 1305 & 105 \\
Metal & 1287 & 40 \\
Sphene & 1270 & \\
Ti oxide & 1250 & 116 \\
Enstatite & 1246 & 109,117 \\
Cr Spinel & 1200 & 115 \\
\hline Amorphous & $<800$ & 40 \\
silicate & & \\
\hline
\end{tabular}

Table 1: Equilibrium condensation temperatures of minerals at $\mathrm{P}=10^{-5}$ bar from solar composition gas [118]. Identified presolar phases are bold. 
conditions of circumstellar environments.

Various search strategies and sample preparations have gradually expanded the inventory of identified presolar grain phases. Many of the most exotic presolar grain types have been found as inclusions within larger grains, principally presolar graphite. Stardust identified in meteorites and IDPs to date includes nanodiamonds, $\mathrm{Si}_{3} \mathrm{~N}_{4}, \mathrm{SiC}$, graphite, $\mathrm{Al}_{2} \mathrm{O}_{3}, \mathrm{TiO}_{2}, \mathrm{Fe}$ oxide, magnetite, hibonite, spinel, $\mathrm{SiO}_{2}$, olivine, and a variety of amorphous silicates. Identified subgrains within these grains include ( $\mathrm{Ti}, \mathrm{Zr}, \mathrm{Mo}, \mathrm{Fe}$ ) carbides, kamacite, metal, $\mathrm{TiO}_{2}$, oldhamite, and silicide [81, 113-117]. These phases include many of the minerals predicted to form by equilibrium condensation from a gas of solar composition in O-rich stellar outflows (Table 1, [118]). Many of these phases have narrow ranges of stability and may react to form other compounds as temperatures decrease before leaving the circumstellar environment. Amorphous silicates condense below about $800 \mathrm{~K}$ [111]. Amorphous silicates may anneal to crystalline phases upon heating to $\sim 1000 \mathrm{~K}$ in a matter of hours to years, depending upon the composition and ambient temperature [111].

\subsection{Origins of GEMS grains}

Twenty years ago, GEMS grains were proposed to be remnants of interstellar silicate grains [59]. This proposal was based on their amorphous structure, size, presence of nanophase $\mathrm{Fe}$, observation of relict internal grains, near-chondritic element abundances, and an infrared spectral match to dust in the ISM. A further prediction was that GEMS grains formed by extensive radiation processing of mineral precursors that condensed in circumstellar outflows [119]. It would be a decade before quantitative tests of this model could be performed, with the introduction of the NanoSIMS ion microprobe and TEM instruments capable of quantitative chemical imaging.

As discussed in the previous section, some GEMS grains do indeed have highly anomalous $\mathrm{O}$ isotopic compositions that show they formed in the outflows of evolved stars. A few other presolar silicates also show evidence of radiation processing after their formation, such as the amorphous enstatite grain discussed above. These observations provide some support for the interstellar GEMS model. However, the chemical and isotopic properties of most GEMS grains are not as expected if they formed by gradual erosion and homogenization of circumstellar dust.

Consequences of this model are that circumstellar dust grains gradually become chemically and isotopically homogenized. At intermediate stages, grains will retain moderate isotopic anomalies. Thoroughly homogenized grains would end up with the average $\mathrm{O}$ isotopic composition of the local ISM and have uniform chemical compositions. However, neither of these predictions is borne out. Excepting the $<6 \%$ of GEMS grains with large isotopic anomalies, the remainder have $\mathrm{O}$ isotopic ratios within error of Solar System materials. The bulk $\mathrm{O}$ isotopic composition of GEMS grains is also within $2 \%$ of terrestrial (or planetary) 


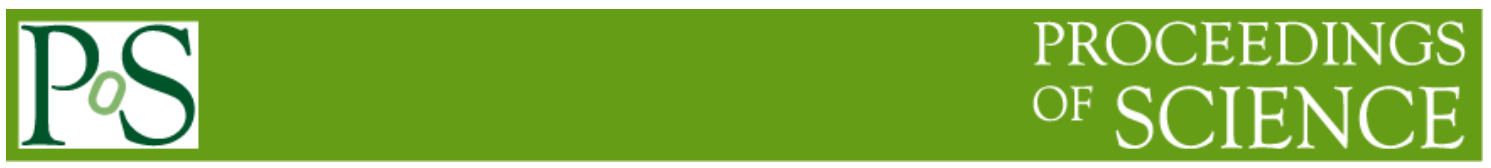

composition. This is expected for planetary materials but the expected end point of such extensive homogenization would actually be the solar value, that is $\sim 5 \%$ more ${ }^{16} \mathrm{O}$ rich than terrestrial composition [120]. While the narrow range of $\mathrm{O}$ isotopic ratios is consistent with thorough homogenization, their chemical compositions are very highly heterogeneous, both within and between GEMS grains (Fig. 7).

A final difficulty for an interstellar origin of GEMS grains is that their bulk elemental abundances differ from compositions derived for interstellar silicate grains based on element depletion patterns in the ISM. Silicates are expected to be the main host for rock-forming elements $(\mathrm{Mg}$,

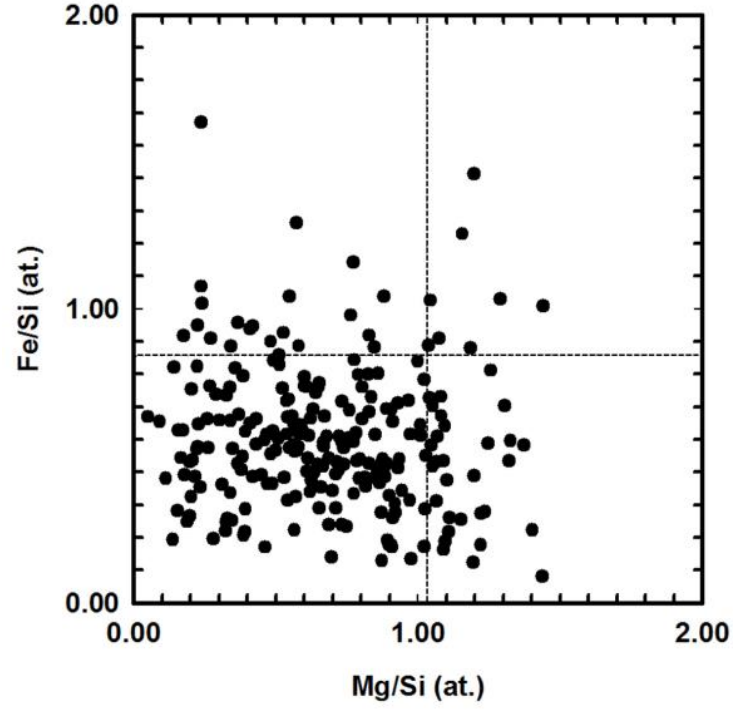

Fig. 7: $\mathrm{Fe} / \mathrm{Si}$ and $\mathrm{Mg} / \mathrm{Si}$ ratios for GEMS grain bulk compositions showing high heterogeneity [60]. The dotted lines are the solar values.

$\mathrm{Fe}, \mathrm{Ca}$ ) that are highly depleted from the gas phase in the ISM. So the expected abundances of these elements in interstellar silicates are near-solar values. However, the average Si-normalized abundances of $\mathrm{Mg}, \mathrm{Fe}$, and $\mathrm{Ca}$ are approximately $60 \%$ of solar values. If GEMS grains did originate in the ISM then a complementary interstellar phase having high $\mathrm{Mg}, \mathrm{Fe}$, and $\mathrm{Ca}$ abundances should be found together with GEMS grains. However, the only such phases occurring in IDPs are crystalline silicates, oxides, and sulfides whose isotopic compositions are indicative of formation within the Solar System [52].

Based on these observations, it has been proposed that most GEMS grains formed in the solar nebula as late stage condensates [60]. The similarity of GEMS grains formed in this way with the O-anomalous circumstellar GEMS grains can be understood if they formed in a similar manner. In fact this is not necessarily surprising because 'dirty' amorphous silicates are the most abundant form of dust produced by evolved O-rich stars, by condensation from gas of near-solar chemical composition. Similar conditions are likely to have been present within the early solar nebula.

Given their high abundance in cometary materials, GEMS grains were clearly one of the major building blocks of outer Solar System materials. GEMS-like materials have also been reported in a few of the most primitive carbonaceous chondrites and some Antarctic micrometeorites [121-124]. The fact that GEMS grains are so much more common in CP-IDPs than meteorites may be attributed to their rapid alteration and destruction by parent body processes, especially aqueous alteration [65]. GEMS-like grains are common in the Stardust mission samples, although their compositions and textures have been thermally modified by the collection process [125]. 


\subsection{Ancient organic dust}

Organic matter is an important component of asteroids and comets and its nature and history is intimately related to disciplines ranging from interstellar chemistry to the origin of life. Early Solar System organic matter has been most intensively studied in carbonaceous chondrite meteorites, where large samples are available for analysis. Meteoritic organic matter is mostly an acid insoluble macromolecular material that is highly aromatic and cross-linked with various functional groups $[126,127]$. Soluble organic molecules only account for about $10 \%$ of the organic component, yet thousands of distinct species have been identified.

The isotopic properties of primitive organic matter give clues to its origin. Organic matter in primitive meteorites and IDPs displays $\mathrm{H}$ and $\mathrm{N}$ isotopic anomalies that are attributed to mass fractionation during chemical reactions at low $\mathrm{T}(10-100 \mathrm{~K})$ in presolar cold molecular clouds or the outer protosolar disk [128-131]. Interstellar origins of D enrichments are supported by observed $\mathrm{D} / \mathrm{H}$ ratios of simple interstellar molecules that reach 10,000 times the local average, in agreement with models of gas phase chemistry in cold molecular clouds [132]. Selective photodissociation of D from PAH molecules in the diffuse ISM is another potential route to Denrichment [133]. The $\mathrm{N}$ isotopic anomalies may require even more extreme conditions. One recent model showed that ${ }^{15} \mathrm{~N}$ enrichments comparable to values in primitive Solar System materials may be produced in amines and nitriles at $10 \mathrm{~K}$ [134]. It has also been proposed that photochemical self-shielding of $\mathrm{N}_{2}$ in the solar nebula led to substantial $\mathrm{N}$ isotopic fractionation [135]. However, recent modeling and experimental efforts have left open the question of whether this process may account for the largest $\mathrm{N}$ isotopic anomalies observed [136, 137].

The magnitudes of $\mathrm{H}$ and $\mathrm{N}$ isotopic anomalies provide a guide to how well presolar organic materials have survived in different materials. CP-IDPs have among the most consistent highly elevated $\mathrm{D} / \mathrm{H}$ and ${ }^{15} \mathrm{~N} /{ }^{14} \mathrm{~N}$ ratios among meteoritic materials [69], perhaps reflecting the fact that these IDPs have escaped hydrothermal processing that has affected meteorites. The largest $\mathrm{H}$ and $\mathrm{N}$ isotopic anomalies in meteorites and IDPs occur in $\mu \mathrm{m}$-sized inclusions [69, 138-141]. Many of these isotopic 'hotspots' are associated with submicrometer, often hollow, organic nanoglobules that are composed of amorphous $\mathrm{C}$ with trace $\mathrm{H}, \mathrm{N}$, and $\mathrm{O}[142,143]$. The globules are isotopically variable and isotopically distinct from the surrounding matrix (Fig. 8). Since parent body processes cannot account for the range of observed $\mathrm{H}, \mathrm{C}$, and $\mathrm{N}$ isotopic anomalies, the organic globules must have formed before their meteorite and IDP parent bodies.

Isotopically anomalous organic nanoglobules have since been identified in many primitive meteorites, CP-IDPs, and comet Wild 2 Stardust mission samples [68, 144-146]. In addition to organic globules, carbonaceous matter in CP-IDPs shows a wide range of morphologies, including bubbly, featureless, and irregular shapes that are all isotopically distinct [147]. Variations in the isotopic signatures and morphologies of organic globules and other forms of anomalous carbonaceous grains likely reflect differing formation conditions and subsequent histories of alteration in the solar nebula or in their parent bodies. Due to their small sizes, little 


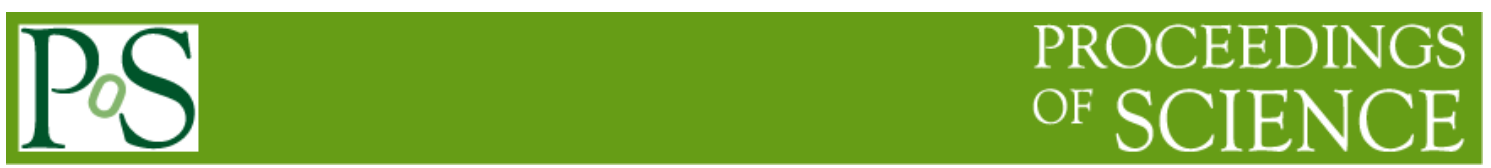

is known about the chemical compositions of the organic globules and what their relationship is to the bulk of the organic matter.

The combined D and ${ }^{15} \mathrm{~N}$ enrichments of organic globules are indicative of low temperature chemical fractionation, but there is some uncertainty in exactly where this occurred. It is known that appropriate conditions are present in cold molecular clouds and it is likely that similar environments were present in the outermost reaches of the protosolar disk. Whatever

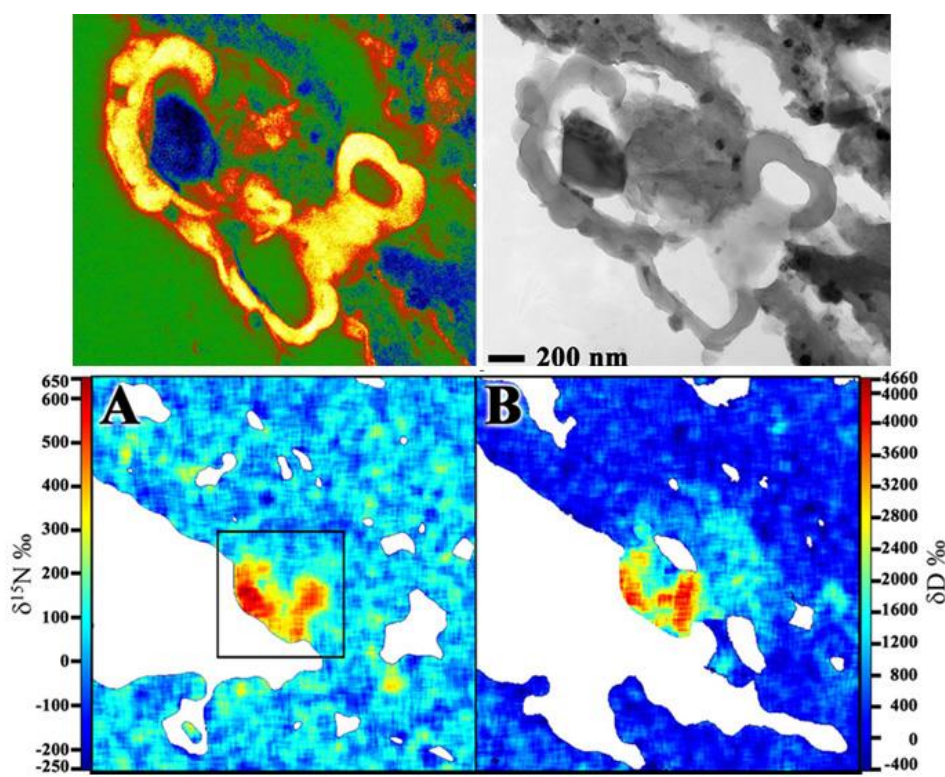

Fig. 8: TEM energy filtered C (top L) and brightfield images (top R) of organic globules in Tagish Lake. The globules are highly enriched in ${ }^{15} \mathrm{~N}$ (lower L) and D (lower R). Black border in A shows the area of the TEM images. Figure from [143].

their origins, the common

observation of organic globules among cometary and asteroidal materials shows they were a significant and widespread constituent of the protoplanetary disk and were an important part of the Solar System starting materials.

\section{Concluding remarks}

The laboratory study of astromaterials has brought about new ways of exploring diverse and remote astrophysical environments. The scientific knowledge gained from the study of cosmic dust has grown exponentially as analytical capabilities have improved and new samples have become available for study. The results of these studies have far reaching implications for the origin of the Solar System and for the study of stellar evolution, interstellar processes and star formation.

For those grains found to have clearly non-solar isotopic ratios, we are afforded a rare and crucial certainty into their origins as stardust grains. This leaves aside any biases or assumptions about what interstellar grains are thought to be like. The ability to definitively identify bona fide stardust grains on the basis of isotopic anomalies has opened up new branches of laboratory astrophysics that were unimaginable a few decades ago. The study of presolar silicates remains a quickly developing scientific frontier. These grains show a breadth of compositions and structures that will both complicate and strengthen new models of dust formation and evolution in the Galaxy. Spectroscopic observations of the ISM provide essential context for understanding how presolar grains fit in with the lifecycle of dust in the Galaxy. An important 
unsettled question is how the processing of dust in the ISM is manifested, either as alteration of existing grains and/or formation of new materials. Continued advances in analytical capabilities in the laboratory and astrophysical communities will undoubtedly bring new perspectives on these issues.

After decades of study, there is still no clear consensus on where micrometeorites and IDPs come from. This is an important question for models of the formation of the planets and the Solar System. These samples have yielded a wealth of information on dozens or even hundreds of Solar System bodies that are at present anonymous. The recent Stardust sample return mission to comet 81P/Wild 2 and Hayabusa mission to asteroid Itokawa marked a new era in Solar System exploration. The study of just a few micrograms of cometary and asteroidal materials obtained directly from their source bodies have both strengthened and overturned longstanding models and have raised new questions that add urgency to continued exploration. Two missions in the planning stages will retrieve much larger surface samples of primitive nearEarth asteroids, the Japanese Hayabusa 2 mission to C-type asteroid 1999 JU3 and the NASA New Frontiers mission to B-type asteroid Bennu [148]. These target asteroids are expected to be primitive and the missions will return pristine samples with well-established geochemical context. A comet surface sample return mission would be an equally exciting target for future missions.

\section{References}

1. McDonnell, J.A.M., et al., Dust particle impacts during the Giotto encounter with Comet GriggSkjellerup. Nature, 1993. 362: p. 732-734.

2. Kissel, J., et al., Composition of comet Halley dust particles from Giotto observations. Nature, 1986. 321: p. 336.

3. Srama, R., et al., The Cassini Cosmic Dust Analyzer. Space Science Reviews, 2004. 114: p. 465518.

4. Krüger, H., et al., Three years of Ulysses dust data: 2005 to 2007. Planetary and Space Science, 2010. 58: p. 951-964.

5. Grün, E., et al., Discovery of Jovian dust streams and interstellar grains by the Ulysses spacecraft. Nature, 1993. 362: p. 428-430.

6. Whipple, F.L., On Maintaining the Meteoritic Complex. SAO Special Report, 1967. 239: p. 1-46.

7. $\quad$ Dermott, S.F., et al., Origin of the solar system dust bands discovered by IRAS. Nature, 1984. 312: p. 505-509.

8. Nesvorný, D., et al., Cometary Origin of the Zodiacal Cloud and Carbonaceous Micrometeorites. Implications for Hot Debris Disks. The Astrophysical Journal, 2010. 713: p. 816-836.

9. Burns, J.A., P.L. Lamy, and S. Soter, Radiation forces on small particles in the solar system. Icarus, 1979. 40: p. 1-48.

10. Robertson, H.P., Dynamical effects of radiation in the solar system. Monthly Notices of the Royal Astronomical Society, 1937. 97: p. 423. 
11. Klačka, J., et al., The Poynting-Robertson effect: A critical perspective. Icarus, 2014. 232: p. 249262.

12. Love, S.G. and D.E. Brownlee, A Direct Measurement of the Terrestrial Mass Accretion Rate of Cosmic Dust. Science, 1993. 262: p. 550-553.

13. Brownlee, D., et al., Comet 81P/Wild 2 Under a Microscope. Science, 2006. 314(5806): p. 17111716.

14. Nakamura, T., et al., Itokawa Dust Particles: A Direct Link Between S-Type Asteroids and Ordinary Chondrites. Science, 2011. 333: p. 1113-1116.

15. Noguchi, T., et al., A chondrule-like object captured by space-exposed aerogel on the international space station. Earth and Planetary Science Letters, 2011. 309: p. 198-206.

16. Matzel, J.E.P., et al., Constraints on the Formation Age of Cometary Material from the NASA Stardust Mission. Science, 2010. 328: p. 483-486.

17. Nakamura, T., et al., Chondrulelike Objects in Short-Period Comet 81P/Wild 2. Science, 2008. 321: p. 1664-1667.

18. McKeegan, K.D., et al., Isotopic Compositions of Cometary Matter Returned by Stardust. Science, 2006. 314(5806): p. 1724-1728.

19. Sandford, S.A., et al., Organics Captured from Comet 81P/Wild 2 by the Stardust Spacecraft. Science, 2006. 314: p. 1720-1724.

20. Landgraf, M., M. Müller, and E. Grün, Prediction of the in-situ dust measurements of the stardust mission to comet 81P/Wild 2. Planetary and Space Science, 1999. 47: p. 1029-1050.

21. Westphal, A.J., et al., Final Reports of the Stardust ISPE: Seven Probable Interstellar Dust Particles, in Lunar and Planetary Science Conference 2014. p. 2269.

22. Noguchi, T., et al., Incipient Space Weathering Observed on the Surface of Itokawa Dust Particles. Science, 2011. 333: p. 1121-1125.

23. Murray, J.K., On the microscopic characters of volcanic ashes and cosmic dust, and their distribution in the deep sea deposits. Proceedings of the Royal Society Edinborough, 1884. 12: p. 474-495.

24. Brownlee, D.E., Cosmic dust: collection and research. Ann. Rev. Earth Planet. Sci., 1985. 13: p. 147-173.

25. Maurette, M., et al., A collection of diverse micrometeorites recovered from 100 tonnes of Antarctic blue ice. Nature, 1991. 351: p. 44-47.

26. Taylor, S., J.H. Lever, and R.P. Harvey, Numbers, types, and compositions of an unbiased collection of cosmic spherules. Meteoritics and Planetary Science, 2000. 35: p. 651-666.

27. Duprat, J., et al., Micrometeorites from Central Antarctic snow: The CONCORDIA collection. Advances in Space Research, 2007. 39: p. 605-611.

28. Genge, M.J., et al., The classification of micrometeorites. Meteoritics and Planetary Science, 2008. 43: p. 497-515.

29. Brearley, A.J. and H.Y. McSween, The Action of Water, in Meteorites and the Early Solar System II, D.S. Lauretta, Editor. 2006. p. 584-624.

30. Kurat, G., et al., Petrology and geochemistry of Antarctic micrometeorites. Geochimica et Cosmochimica Acta, 1994. 58: p. 3879-3904.

31. Duprat, J., et al., Extreme deuterium excesses in ultracarbonaceous micrometeorites from central Antarctic snow. Science, 2010. 328(5979): p. 742-5.

32. Brownlee, D.E., P.W. Hodge, and W. Bucher, The Physical Nature of Interplanetary Dust as Inferred by Particles Collected at 35 KM. NASA Special Publication, 1973. 319: p. 291-295.

33. Sandford, S.A., The collection and analysis of extraterrestrial dust particles. Fundamentals of Cosmic Physics, 1987. 12(1): p. 73.

34. Messenger, S., et al., Pristine Stratospheric Collection of Cosmic Dust, in Lunar and Planetary Science Conference2012. p. 2696.

35. Brownlee, D.E., et al., An Atlas of extraterrestrial particles collected with NASA U-2 aircraft, 1974 - 1976. NASA STI/Recon Technical Report, 1976. 76. 
36. Bradley, J.P., D.E. Brownlee, and P. Fraundorf, Discovery of nuclear tracks in interplanetary dust. Science, 1984. 226: p. 1432-1434.

37. Zinner, E., K.D. McKeegan, and R.M. Walker, Laboratory measurements of D/H ratios in interplanetary dust. Nature, 1983. 305: p. 119-121.

38. McKeegan, K.D., Oxygen isotopes in refractory stratospheric dust particles: proof of extraterrestrial origin. Science, 1987. 237: p. 1468-1471.

39. Stadermann, F.J., R.M. Walker, and E. Zinner, Ion microprobe measurements of nitrogen and carbon isotopic variations in individual IDPs. Meteoritics, 1989. 24: p. 327.

40. Messenger, S., et al., Samples of stars beyond the solar system: Silicate grains in interplanetary dust. Science, 2003. 300: p. 105-108.

41. Brownlee, D.E., E. Olszewski, and M. Wheelock, A Working Taxonomy for Micrometeorites, in Lunar and Planetary Institute Science Conference 1982. p. 71-72.

42. Germani, M.S., J.P. Bradley, and D.E. Brownlee, Automated thin-film analyses of hydrated interplanetary dust particles in the analytical electron microscope. Earth and Planetary Science Letters, 1990. 101: p. 162-179.

43. Bradley, J.P. and D.E. Brownlee, An interplanetary dust particle linked directly to type CM meteorites and an asteroidal origin. Science, 1991. 251: p. 549-552.

44. Keller, L.P., K.L. Thomas, and D.S. McKay, An interplanetary dust particle with links to CI chondrites. Geochimica et Cosmochimica Acta, 1992. 56: p. 1409-1412.

45. Rietmeijer, F.J.M., CM-like interplanetary dust particles in lower stratosphere during 1989 October and 1991 June/July. Meteoritics and Planetary Science, 1996. 31: p. 278-288.

46. Nakamura, K., et al., Mineralogical Study of Hydrated IDPs: X-Ray Diffraction and Transmission Electron Microscopy, in Lunar and Planetary Institute Science Conference Abstracts, S. Mackwell, Editor 2004. p. 1862.

47. Thomas, K.L., et al., High Carbon Abundances in IDPs: Hydrated Particles from Cometary Sources? Meteoritics, 1992. 27: p. 296.

48. Keller, L.P., K.L. Thomas, and D.S. McKay, Carbon abundances, major element chemistry, and mineralogy of hydrated interplanetary dust particles, in Lunar and Planetary Institute Science Conference Abstracts1993. p. 785-786.

49. Snead, C.J., et al., Mineralogy and Oxygen Isotope Compositions of Two C-Rich Hydrated Interplanetary Dust Particles, in Meteoritics and Planetary Science Supplement2012. p. 5378.

50. Zolensky, M.E. and K.L. Thomas, Iron and iron-nickel sulfides in chondritic interplanetary dust particles. Geochimica et Cosmochimica Acta, 1995. 59: p. 4707-4712.

51. Thomas, K.L., et al., Carbon abundance and silicate mineralogy of anhydrous interplanetary dust particles. Geochimica et Cosmochimica Acta, 1993. 57(7): p. 1551-1566.

52. Keller, L.P., et al., The Nature and Origin of Interplanetary Dust: High-Temperature Components, in Chondrites and the Protoplanetary Disk, A.N. Krot, Editor 2005. p. 657.

53. Klock, W., et al., Unusual olivine and pyroxene composition in interplanetary dust and unequilibrated ordinary chondrites. Nature, 1989. 339: p. 126-128.

54. Lodders, K., Solar system abundances and condensation temperatures of the elements. Astrophys. J., 2003. 591: p. 1220-1247.

55. Bradley, J.P., D.E. Brownlee, and D.R. Veblen, Pyroxene whiskers and platelets in interplanetary dust - Evidence of vapour phase growth. Nature, 1983. 301: p. 473-477.

56. Bradley, J.P., Nanometer-scale mineralogy and petrography of fine-grained aggregates in anhydrous interplanetary dust particles. Geochimica et Cosmochimica Acta, 1994. 58: p. 21232134.

57. Keller, L.P. and S. Messenger, Equilibrated Aggregates in Cometary IDPs: Insights into the Crystallization Process in Protoplanetary Disks, in Lunar and Planetary Institute Science Conference Abstracts2009. p. 2121.

58. Brownlee, D.E., et al., Cooked GEMS - Insights into the hot origin of crystalline silicates in circumstellar disks and the cold origin GEMS. Lunar \& Planetary Science, 2005. XXXVI: p. 2391. 
59. Bradley, J.P., Chemically anomalous, preaccretionally irradiated grains in interplanetary dust from comets. Science, 1994. 265: p. 925-929.

60. Keller, L.P. and S. Messenger, On the origins of GEMS grains. Geochim. Cosmochim. Acta, 2011. 75: p. 5336-5365.

61. Dai, Z.R. and J.P. Bradley, Iron-nickel sulfides in anhydrous interplanetary dust particles. Geochim. Cosmochim. Acta, 2001. 65: p. 3601-3612.

62. Christoffersen, R. and P.R. Buseck, Mineralogy of interplanetary dust particles from the 'olivine' infrared class. Earth and Planetary Science Letters, 1986. 78: p. 53-66.

63. Keller, L.P., et al., GEMS Revealed: Spectrum Imaging of Aggregate Grains in Interplanetary Dust, in 36th Annual Lunar and Planetary Science Conference, S. Mackwell, Editor 2005. p. 2088.

64. Lauretta, D.S., K. Lodders, and B. Fegley, Jr., Kamacite Sulfurization in the Solar Nebula. Meteoritics and Planetary Science, 1998. 33: p. 821.

65. Nakamura-Messenger, K., et al., Experimental aqueous alteration of cometary dust. Meteoritics \& Planetary Science, 2011. 46(6): p. 843-856.

66. Bradley, J.P. and D.E. Brownlee, Cometary particles: thin sectioning and electron beam analysis. Science (New York, N.Y.), 1986. 231(4745): p. 1542-4.

67. Rietmeijer, F.J.M. and I.D.R. Mackinnon, Cometary evolution : Clues from chondritic interplanetary dust particles, in Proceedings of the International Symposium on the Diversity and Similarity of Comets, E.J. Rolfe and B. Battrick, Editors. 1987, European Space Agency: Brussels, Belgium. p. 363-367.

68. Busemann, H., et al., Ultra-primitive interplanetary dust particles from the comet 26P/GriggSkjellerup dust stream collection. Earth \& Planet. Sci. Lett., 2009. 288: p. 44-57.

69. Messenger, S., Identification of molecular-cloud material in interplanetary dust particles. Nature, 2000. 404: p. 968-971.

70. Floss, C., et al., Identification of isotopically primitive interplanetary dust particles: A NanoSIMS isotopic imaging study. Geochim. Cosmochim. Acta, 2006. 70: p. 2371-2399.

71. Berger, E.L., et al., Evidence for aqueous activity on comet 81P/Wild 2 from sulfide mineral assemblages in Stardust samples and CI chondrites. Geochimica et Cosmochimica Acta, 2011. 75 : p. 3501-3513.

72. Sandford, S.A., Constraints on the Parent Bodies of Collected Interplanetary Dust Particles, in Origin and Evolution of Interplanetary Dust, A.C. Levasseur-Regourd and H. Hasegawa, Editors. 1991, Springer Netherlands. p. 397-404.

73. Thomas, K.L., et al., An asteroidal breccia: The anatomy of a cluster IDP. Geochim. Cosmochim. Acta, 1995. 59: p. 2797-2815.

74. Brownlee, D.E., et al., Identification of Individual Cometary IDP's by Thermally Stepped He Release, in Lunar and Planetary Institute Science Conference Abstracts 1995. p. 183.

75. Flynn, G.J., The near-Earth enhancement of asteroidal over cometary dust. Lunar Planet. Sci., 1990. XXI: p. 363-371.

76. Bradley, J.P., S.A. Sandford, and R.M. Walker, Interplanetary dust particles, in Meteorites and the Early Solar System, J.F. Kerridge and M.S. Matthews, Editors. 1988, University of Arizona Press. p. 861-895.

77. Messenger, S., et al., The Population of Starting Materials Available for Solar System Construction, in Meteorites and the Early Solar System II, D.S. Lauretta, Editor. 2006. p. 187-208.

78. Astrophysical Implications of the Laboratory Study of Presolar Materials. AIP Conference Proceedings. 1998: American Institute of Physics.

79. Clayton, D.D. and L.R. Nittler, Astrophysics with presolar stardust. Ann. Rev. Astron. Astrophys., 2004. 42: p. 39-78.

80. Davis, A.M., Cosmochemistry Special Feature: Stardust in meteorites. Proceedings of the National Academy of Science, 2011. 108: p. 19142-19146.

81. Zinner, E., 1.4 - Presolar Grains, in Treatise on Geochemistry (Second Edition), H.D. Holland and K.K. Turekian, Editors. 2014, Elsevier: Oxford. p. 181-213.

82. Amari, S., et al., Interstellar graphite in meteorites. Nature, 1990. 345: p. 238-240. 
83. Lewis, R.S., et al., Interstellar diamonds in meteorites. Nature, 1987. 326: p. 160-162.

84. Bernatowicz, T., et al., Evidence for interstellar SiC in the Murray carbonaceous meteorite. Nature, 1987. 330: p. 728-730.

85. Hynes, K.M. and F. Gyngard, The Presolar Grain Database: http://presolar.wustl.edu/ pgd. Lunar \& Planetary Science, 2009. XL: p. Abstract \#1198.

86. Nittler, L.R., et al., Extinct ${ }^{44} \mathrm{Ti}$ in presolar graphite and SiC: Proof of a supernova origin. Astrophys. J., 1996. 462: p. L31-L34.

87. Woosley, S.E. and T.A. Weaver, The evolution and explosion of massive stars, II. Explosive hydrodynamics and nucleosynthesis. Astrophys. J. Suppl., 1995. 101: p. 181-235.

88. Bernatowicz, T.J., et al., Pristine presolar silicon carbide. Geochim. Cosmochim. Acta, 2003. 67: p. 4679-4691.

89. Amari, S., E. Zinner, and R. Gallino, Presolar graphite from the Murchison Meteorite: An isotopic study. Geochimica et Cosmochimica Acta, 2014. 133(0): p. 479-522.

90. Bernatowicz, T.J., et al., Interstellar grains within interstellar grains. The Astrophysical Journal Letters, 1991. 373: p. L73-L76.

91. Bernatowicz, T.J., et al., Constraints on grain formation around carbon stars from laboratory studies of presolar graphite. Astrophys. J., 2005. 631: p. 988-1000.

92. Croat, T.K., F.J. Stadermann, and T.J. Bernatowicz, Presolar graphite from AGB stars: Microstructure and s-process enrichment. Astrophys. J., 2005. 631: p. 976-987.

93. Messenger, S., et al., Indigenous polycyclic aromatic hydrocarbons in circumstellar graphite grains from primitive meteorites. Astrophys. J., 1998. 502: p. 284-295.

94. Daulton, T.L., et al., Genesis of presolar diamonds: Comparative high-resolution transmission electron microscopy study of meteoritic and terrestrial nano-diamonds. Geochim. Cosmochim. Acta, 1996. 60: p. 4853-4872.

95. Marty, B., et al., A 15N-Poor Isotopic Composition for the Solar System As Shown by Genesis Solar Wind Samples. Science, 2011. 332: p. 1533-1536.

96. Hutcheon, I.D., et al., Extreme ${ }^{26} \mathrm{Mg}$ and ${ }^{17} \mathrm{O}$ enrichments in an Orgueil corundum: Identification of a presolar oxide grain. Astrophys. J., 1994. 425: p. L97-L100.

97. Nittler, L.R., et al., Identification of an interstellar oxide grain from the Murchison meteorite by ion imaging. Lunar Planet. Sci., 1993. XXIV: p. 1087-1088.

98. Nittler, L.R., et al., Stellar sapphires: The properties and origins of presolar $\mathrm{Al}_{2} \mathrm{O}_{3}$ in meteorites. Astrophys. J., 1997. 483: p. 475-495.

99. Nguyen, A.N. and E. Zinner, Discovery of ancient silicate stardust in a meteorite. Science, 2004. 303: p. 1496-1499.

100. Yurimoto, H., K. Nagashima, and T. Kunihiro, High precision isotope micro-imaging of materials. Appl. Surf. Sci., 2003. 203-204: p. 793-797.

101. Molster, F.J., et al., Crystalline silicate dust around evolved stars. III. A correlations study of crystalline silicate features. Astron. \& Astrophys., 2002. 382: p. 241-255.

102. Kemper, F., W.J. Vriend, and A.G.G.M. Tielens, The absence of crystalline silicates in the diffuse interstellar medium. Astrophys. J., 2004. 609: p. 826-837.

103. Rho, J., et al., Spitzer Observations of the Young Core-Collapse Supernova Remnant 1E0102-72.3: Infrared Ejecta Emission and Dust Formation. Astrophys. J., 2009. 700(1): p. 579-596.

104. Evans, A., et al., Infrared spectroscopy of Nova Cassiopeiae 1993 - IV. A closer look at the dust. Mon. Not. R. Astron. Soc., 2005. 360(4): p. 1483-1492.

105. Messenger, S., L.P. Keller, and D.S. Lauretta, Supernova olivine from cometary dust. Science, 2005. 309: p. 737-741.

106. Ferrarotti, A.S. and H.-P. Gail, Mineral formation in stellar winds II: Effects of Mg/Si abundance variations on dust composition in AGB stars. Astron. Astrophy., 2001. 371: p. 133-151.

107. Messenger, S., et al., History of nebular processing traced by silicate stardust in IDPs, in Lunar \& Planetary Science2010. p. Abstract \#2483.

108. Vollmer, C., et al., Stellar MgSiO3 perovskite: a shock-transformed stardust silicate found in a meteorite. Astrophy. J., 2007. 666: p. L49-L52. 
109. Nguyen, A.N., et al., Microstructure of a supernova silicate grain, in Meteorit. Planet. Sci.2010. p. A5423.

110. Nguyen, A.N., et al., Crystal Structure and Chemical Composition of a Presolar Silicate from the Queen Elizabeth Range 99177 Meteorite, in Lunar and Planetary Science Conference2013. p. 2853.

111. Gail, H.-P., Formation and Evolution of Minerals in Accretion Disks and Stellar Outflows, in Astromineralogy, T. Henning, Editor. 2003, Springer. p. 55-120.

112. Nguyen, A.N., et al., Identification of a Compound Spinel and Silicate Presolar Grain in a Chondritic Interplanetary Dust Particle, in Lunar and Planetary Science Conference2014. p. 2351.

113. Choi, B.-G., G.J. Wasserburg, and G.R. Huss, Circumstellar hibonite and corundum and nucleosynthesis in asymptotic giant branch stars. Astrophys. J., 1999. 522: p. L133-L136.

114. Zega, T.J., et al., A transmission electron microscopy study of presolar spinel. Geochimica et Cosmochimica Acta, 2014. 124: p. 152-169.

115. Zinner, E., et al., Oxygen, magnesium and chromium isotopic ratios of presolar spinel grains. Geochim. Cosmochim. Acta, 2005. 69: p. 4149-4165.

116. Nittler, L.R. and C.M.O.D. Alexander, Automatic identification of presolar Al- and Ti-rich oxide grains from ordinary chondrites. Lunar Planet. Sci., 1999. XXX: p. Abstract \#2041.

117. Vollmer, C., P. Hoppe, and F.E. Brenker, Transmission Electron Microscopy of Al-rich Silicate Stardust from Asymptotic Giant Branch Stars. The Astrophysical Journal, 2013. 769: p. 61.

118. Yoneda, S. and L. Grossman, Condensation of $\mathrm{CaO}-\mathrm{MgO}-\mathrm{Al}_{2} \mathrm{O}_{3}-\mathrm{SiO}_{2}$ liquids from cosmic gases. Geochim. Cosmochim. Acta, 1995. 59: p. 3413-3444.

119. Bradley, J.P. and Z.R. Dai, Mechanism of formation of glass with embedded metal and sulfides. Astrophys. J., 2004. 617: p. 650-655.

120. McKeegan, K.D., et al., The Oxygen Isotopic Composition of the Sun Inferred from Captured Solar Wind. Science, 2011. 332: p. 1528-1532.

121. Keller, L.P., K. Nakamura-Messenger, and S. Messenger, Amorphous silicates in primitive meteoritic materials: Acfer 094 and IDPs, in Meteorit. Planet. Sci.2009. p. A5371.

122. Dobrică, E., et al., Transmission Electron Microscopy of CONCORDIA UltraCarbonaceous Antarctic MicroMeteorites (UCAMMs): Mineralogical properties. Geochimica et Cosmochimica Acta, 2012. 76: p. 68-82.

123. Keller, L.P. and S. Messenger, Formation and Processing of Amorphous Silicates in Primitive Carbonaceous Chondrites and Cometary Dust, in Lunar and Planetary Science Conference 2012. p. 1880.

124. Leroux, H., et al., Sub-Micrometer Composition Fields of Acfer 094 and Paris Matrices, in Lunar and Planetary Science Conference2014. p. 1706.

125. Leroux, H. and D. Jacob, Fine-grained material encased in microtracks of Stardust samples. Meteoritics and Planetary Science, 2013. 48: p. 1607-1617.

126. Cronin, J.R., S. Pizzarello, and D.P. Cruikshank, Organic matter in carbonaceous chondrites, planetary satellites, asteroids and comets, in Meteorites and the Early Solar System, J.F. Kerridge and M.S. Matthews, Editors. 1988, The Univ. of Ariz. Press: Tucson.

127. Pizzarello, S., G.W. Cooper, and G.J. Flynn, The Nature and Distribution of the Organic Material in Carbonaceous Chondrites and Interplanetary Dust Particles, in Meteorites and the Early Solar System II, D.S. Lauretta and H.Y. McSween, Editors. 2006, University of Arizona Press. p. 625651.

128. Aikawa, Y. and E. Herbst, Deuterium Fractionation in Protoplanetary Disks. The Astrophysical Journal, 1999. 526: p. 314-326.

129. Geiss, J. and H. Reeves, Deuterium in the solar system. Astron. Astrophys., 1981. 93: p. 189-199.

130. Messenger, S. and R. Walker, Evidence for molecular cloud material in meteorites and interplanetary dust, in Astrophysical Implications of the Laboratory Study of Presolar Materials, T. Bernatowicz and E. Zinner, Editors. 1997, AIP: New York. p. 545-566.

131. Zinner, E., Interstellar cloud material in meteorites, in Meteorites and the Early Solar System, J.F. Kerridge and M.S. Matthews, Editors. 1988, University of Arizona Press: Tucson. p. 956-983. 
132. Millar, T.J., A. Bennett, and E. Herbst, Deuterium fractionation in dense interstellar clouds. The Astrophysical Journal, 1989. 340: p. 906-920.

133. Sandford, S.A., M.P. Bernstein, and J.P. Dworkin, Assessment of the interstellar processes leading to deuterium enrichment in meteoritic organics. Meteorit. Planet. Sci., 2001. 36: p. 1117-1133.

134. Rodgers, S.D. and S.B. Charnley, Nitrogen Isotopic Fractionation of Interstellar Nitriles. The Astrophysical Journal, 2008. 689: p. 1448-1455.

135. Clayton, R.N., Self-shielding in the solar nebula. Nature, 2002. 415: p. 860-861.

136. Lyons, J.R., Isotope Signatures in Organics due to $\mathrm{CO}$ and $N_{-} 2$ Self-Shielding, in Lunar and Planetary Science Conference2012. p. 2858.

137. Chakraborty, S., et al., Huge Isotope Effect in VUV Photodissociation of N_2: Implications for Meteorite Data, in Lunar and Planetary Science Conference2013. p. 1043.

138. McKeegan, K.D., et al., Hydrogen isotopic variations in interplanetary dust particles. Lunar Planet. Sci., 1987. XVIII: p. 627-628.

139. Busemann, H., et al., Interstellar chemistry recorded in organic matter from primitive meteorites. Science, 2006. 312: p. 727-730.

140. Floss, C., et al., Carbon and nitrogen isotopic anomalies in an anhydrous interplanetary dust particle. Science, 2004. 303: p. 1355-1358.

141. Messenger, S., et al., Isotopic signatures of presolar materials in interplanetary dust. Space Sci. Rev., 2003. 106: p. 155-172.

142. Nakamura, K., et al., Hollow organic globules in the Tagish Lake meteorite as possible products of primitive organic reactions. International Journal of Astrobiology, 2002. 1: p. 179-189.

143. Nakamura-Messenger, K., et al., Organic globules in the Tagish Lake meteorite: remnants of the protosolar disk. Science, 2006. 314: p. 1439-1442.

144. Messenger, S., K. Nakamura-Messenger, and L.P. Keller, 15N-rich Organic Globules in a Cluster IDP and the Bells CM2 Chondrite, in Lunar and Planetary Science Conference2008. p. 2391.

145. Matrajt, G., et al., Carbon investigation of two Stardust particles: A TEM, NanoSIMS, and XANES study. Meteoritics and Planetary Science, 2008. 43: p. 315-334.

146. De Gregorio, B.T., et al., Isotopic anomalies in organic nanoglobules from Comet 81P/Wild 2: Comparison to Murchison nanoglobules and isotopic anomalies induced in terrestrial organics by electron irradiation. Geochimica et Cosmochimica Acta, 2010. 74: p. 4454-4470.

147. Matrajt, G., et al., Diverse forms of primordial organic matter identified in interplanetary dust particles. Meteoritics \& Planetary Science, 2012. 47(4): p. 525-549.

148. Lauretta, D.S., et al., The OSIRIS-REx Mission - Sample Acquisition Strategy and Evidence for the Nature of Regolith on Asteroid (101955) 1999 RQ36. LPI Contributions, 2012. 1667: p. 6291. 